\title{
Optimal Remote State Estimation for Self-Propelled Particle Models
}

\author{
Shinkyu Park and Nuno C. Martins
}

\begin{abstract}
We investigate the design of a remote state estimation system for a self-propelled particle (SPP). Our framework consists of a sensing unit that accesses the full state of the SPP and an estimator that is remotely located from the sensing unit. The sensing unit must pay a cost when it chooses to transmit information on the state of the SPP to the estimator; and the estimator computes the best estimate of the state of the SPP based on received information. In this paper, we provide methods to design transmission policies and estimation rules for the sensing unit and estimator, respectively, that are optimal for a given cost functional that combines state estimation distortion and communication costs. We consider two notions of optimality: joint optimality and person-by-person optimality 1 Our main results show the existence of a jointly optimal solution and describe an iterative procedure to find a person-by-person optimal solution. In addition, we explain how the remote estimation scheme can be applied to tracking of animal movements over a costly communication link. We also provide experimental results to show the effectiveness of the scheme.
\end{abstract}

\section{INTRODUCTION}

Consider a self-propelled particle (SPP) moving in a two-dimensional plane whose state $\mathbf{x}_{k}$ is represented as follows:

$$
\mathbf{x}_{k}=\left(\begin{array}{lll}
\mathbf{p}_{1, k} & \mathbf{p}_{2, k} & \boldsymbol{\theta}_{k}
\end{array}\right)^{T} \in \mathbb{R}^{2} \times[0,2 \pi)
$$

where $\left(\mathbf{p}_{1, k}, \mathbf{p}_{2, k}\right)$ and $\boldsymbol{\theta}_{k}$ represent the location in the plane and the orientation at time $k$, respectively. The state of the SPP evolves according to the following model:

$$
\left(\begin{array}{c}
\mathbf{p}_{1, k+1} \\
\mathbf{p}_{2, k+1} \\
\boldsymbol{\theta}_{k+1}
\end{array}\right)=\left(\begin{array}{c}
\mathbf{p}_{1, k}+\mathbf{v}_{k} \cos \left(\boldsymbol{\theta}_{k}+\boldsymbol{\phi}_{k}\right) \\
\mathbf{p}_{2, k}+\mathbf{v}_{k} \sin \left(\boldsymbol{\theta}_{k}+\boldsymbol{\phi}_{k}\right) \\
\boldsymbol{\theta}_{k}+\boldsymbol{\phi}_{k}
\end{array}\right), k \geq 0
$$

with the initial condition $\mathbf{x}_{0}=x_{0}=\left(\begin{array}{lll}p_{1,0} & p_{2,0} & \theta_{0}\end{array}\right)^{T}$. The random processes $\mathbf{v}_{k}$ and $\phi_{k}$ represent the translational and angular velocities, respectively.

In this paper, we consider a remote estimation system formed by a sensing unit and remotely located estimator: The sensing unit accesses $\mathbf{x}_{k}$ and has the authority to decide whether to transmit it to the estimator. The sequence

Shinkyu Park and Nuno C. Martins are with the Department of Electrical and Computer Engineering, University of Maryland College Park, College Park, MD 20742-4450, USA. \{skpark, nmartins\}@umd.edu

${ }^{1}$ The precise definitions of joint optimality and person-by-person optimality are given in Definition II.2 and Definition II.3 respectively. 
of decisions on whether to transmit is represented by $\mathbf{R}_{k}$, for which $\mathbf{R}_{k}=1$ if the sensing unit decides to transmit and $\mathbf{R}_{k}=0$ otherwise. The cost of each transmission is represented by $c_{k}$. The estimator computes a state estimate

$\hat{\mathbf{x}}_{k}=\left(\begin{array}{lll}\hat{\boldsymbol{p}}_{1, k} & \hat{\boldsymbol{p}}_{2, k} & \hat{\boldsymbol{\theta}}_{k}\end{array}\right)^{T}$ based on received information. The diagram in Fig. 1 depicts the overall framework adopted here.

\section{A. Outline of Main Results}

Let a transmission policy $\mathcal{T}_{k}$ and an estimation rule $\mathcal{E}_{k}$ for the sensing unit and estimator at time $k$, respectively, be defined as follows:

$$
\begin{aligned}
\mathcal{T}_{k} & :\left(\mathbb{R}^{2} \times[0,2 \pi)\right)^{k+1} \times\{0,1\}^{k-1} \rightarrow\{0,1\} \\
& \mathcal{E}_{k}:\left(\mathbb{R}^{2} \times[0,2 \pi)\right)^{\left|\mathcal{I}^{k}\right|+1} \times\{0,1\}^{k} \rightarrow \mathbb{R}^{2} \times[0,2 \pi)
\end{aligned}
$$

where the variable $\mathcal{I}^{k}=\left\{\mathbf{x}_{j} \mid \mathbf{R}_{j}=1,1 \leq j \leq k\right\}$ represents information transmitted to the estimator up to time $k$.

Our main goal is to obtain methods to design transmission policies $\left(\mathcal{T}_{1}, \cdots, \mathcal{T}_{N}\right)$ and estimation rules $\left(\mathcal{E}_{1}, \cdots, \mathcal{E}_{N}\right)$ that are optimal for the following cost functional:

$$
\begin{aligned}
& \mathcal{J}\left(x_{0},\left(\mathcal{T}_{1}, \cdots, \mathcal{T}_{N}\right),\left(\mathcal{E}_{1}, \cdots, \mathcal{E}_{N}\right)\right) \\
& =\sum_{k=1}^{N} \mathbb{E}\left[d^{2}\left(\mathbf{x}_{k}, \hat{\mathbf{x}}_{k}\right)+c_{k} \cdot \mathbf{R}_{k} \mid \mathbf{x}_{0}=x_{0},\left(\mathcal{T}_{1}, \cdots, \mathcal{T}_{N}\right),\left(\mathcal{E}_{1}, \cdots, \mathcal{E}_{N}\right)\right]
\end{aligned}
$$

subject to the SPP model 11 and

$$
\begin{aligned}
\mathbf{R}_{k} & =\mathcal{T}_{k}\left(\left(\mathbf{x}_{0}, \cdots, \mathbf{x}_{k}\right),\left(\mathbf{R}_{1}, \cdots, \mathbf{R}_{k-1}\right)\right) \\
\hat{\mathbf{x}}_{k} & =\mathcal{E}_{k}\left(\left(\mathbf{x}_{0}, \mathcal{I}^{k}\right),\left(\mathbf{R}_{1}, \cdots, \mathbf{R}_{k}\right)\right)
\end{aligned}
$$

for each $k$ in $\{1, \cdots, N\}$, where we use Frobenius norm to define the metric $d$ as follows:

$$
d\left(x_{k}, \hat{x}_{k}\right)=\left\|\left(\begin{array}{ccc}
\cos \theta_{k} & -\sin \theta_{k} & p_{1, k} \\
\sin \theta_{k} & \cos \theta_{k} & p_{2, k} \\
0 & 0 & 1
\end{array}\right)-\left(\begin{array}{ccc}
\cos \hat{\theta}_{k} & -\sin \hat{\theta}_{k} & \hat{p}_{1, k} \\
\sin \hat{\theta}_{k} & \cos \hat{\theta}_{k} & \hat{p}_{2, k} \\
0 & 0 & 1
\end{array}\right)\right\|_{F}
$$

Our problem is non-trivial because (2) is in general non-convex and searching for a solution that achieves the minimum over a function space is computationally complex. We adopt a team decision framework in which the sensing unit and the estimator are viewed as players. The following are our main contributions:

1) First, we show that there is a jointly optimal solution which minimizes the cost functional (2). As joint optimality implies person-by-person optimality, this result ensures that the set of person-by-person optimal solutions is non-empty.

2) We propose an iterative procedure, which is inspired by Lloyd's algorithm [1], to compute a person-by-person optimal solution. The procedure alternates between finding the best transmission policies for (2) with the estimation rules fixed, and vice versa; and it generates a sequence of sub-optimal solutions. Our analysis 


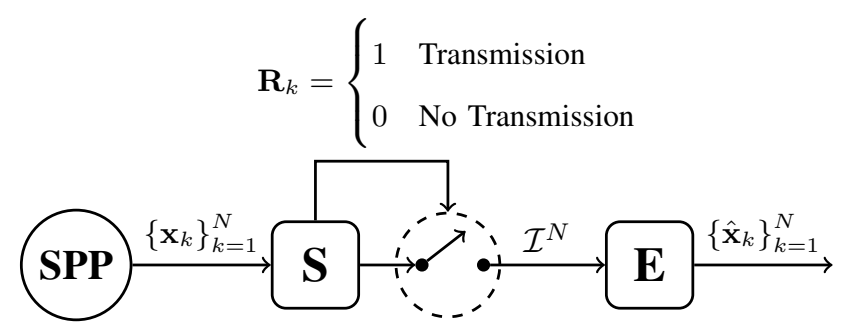

Fig. 1. A remote estimation framework comprised of a self-propelled particle (SPP), a sensing unit (S), and an estimator (E), where $\mathcal{I}^{N}=\left\{\mathbf{x}_{k} \mid \mathbf{R}_{k}=1,1 \leq k \leq N\right\}$.

will show that the sequence has a convergent subsequence; and the limit of any convergent subsequence is a person-by-person optimal solution.

3) We illustrate the performance of the optimal remote estimation scheme in the context of tracking of animal movements over a costly communication link. Our numerical results use GPS data collected from a monitoring device mounted on an African buffalo.

\section{B. Paper Organization}

In Section II] we describe the problem formulation considered throughout the paper, and briefly describe our methodology to find a solution. The main strategy is to decompose the problem into sub-problems, which we can solve sequentially. In Section III, we examine the existence of a jointly optimal solution to each sub-problem. We also describe an iterative procedure for finding a person-by-person optimal solution. Section IV discusses an application of our results to tracking of animal movements and also presents experimental results.

\section{Problem Formulation}

\section{A. Notation and Terminology}

- For a finite sequence of elements $a_{1}, \cdots, a_{N}$ belonging to a set, we adopt the shorthand notation $a_{1: N}=\left(a_{1}, \cdots, a_{N}\right)$.

- For a finite sequence of functions $\mathcal{A}_{1}, \cdots, \mathcal{A}_{N}$ defined on a set, we adopt the shorthand notation $\mathcal{A}_{1: N}=\left(\mathcal{A}_{1}, \cdots, \mathcal{A}_{N}\right)$.

- For $\left\{\mathbf{R}_{j}\right\}_{j=1}^{k-1}$, we define 2

$$
\boldsymbol{\tau}_{k}=\max \left\{1 \leq j \leq k-1 \mid \mathbf{R}_{j}=1\right\}
$$

We refer to $\tau_{k}$ as the last transmission time before time $k$.

${ }^{2}$ We adopt a convention that $\boldsymbol{\tau}_{k}=0$ if $\mathbf{R}_{j}=0$ for all $j$ in $\{1, \cdots, k-1\}$. 


\section{B. Problem Description}

We start by assuming that transmission policies and estimation rules have the following structur ${ }^{3}$. The transmission policy, which may be randomized 4 at time $k$ depends on the last transmission time $\boldsymbol{\tau}_{k}$, the information $\mathbf{x}_{\tau_{k}}$ transmitted to the estimator at time $\tau_{k}$, and the current state $\mathbf{x}_{k}$ of the SPP. The estimation rule at time $k$ depends on the last transmission time $\tau_{k}$ and the information $\mathbf{x}_{\tau_{k}}$ received from the sensing unit at time $\boldsymbol{\tau}_{k}$.

According to (3) and the structural assumptions mentioned above, the variable $\mathbf{R}_{k}$ and estimate $\hat{\mathbf{x}}_{k}$ are determined by a transmission policy $\mathcal{T}_{k}$ and an estimation rule $\mathcal{E}_{k}$ as follows:

$$
\begin{aligned}
\mathbf{R}_{k} & =\mathcal{T}_{k}\left(\boldsymbol{\tau}_{k}, \mathbf{x}_{\boldsymbol{\tau}_{k}}, \mathbf{x}_{k}\right) \\
\hat{\mathbf{x}}_{k} & = \begin{cases}\mathcal{E}_{k}\left(\boldsymbol{\tau}_{k}, \mathbf{x}_{\boldsymbol{\tau}_{k}}\right) & \text { if } \mathbf{R}_{k}=0 \\
\mathbf{x}_{k} & \text { otherwise }\end{cases}
\end{aligned}
$$

We formally state our main problem as follows.

Problem II.1: Find transmission policies $\mathcal{T}_{1: N}$ and estimation rules $\mathcal{E}_{1: N}$ that are optimal for the cost functional (2) subject to the SPP model (11) with the initial condition $\mathrm{x}_{0}=x_{0}$ and (4) 5

We consider the following two notions of optimality for Problem II.1

Definition II.2: We say that transmission policies $\mathcal{T}_{1: N}^{*}$ and estimation rules $\mathcal{E}_{1: N}^{*}$ are jointly optimal for (2) if they achieve the global minimum for every $x_{0}$ in $\mathbb{R}^{2} \times[0,2 \pi)$.

Definition II.3: We say that transmission policies $\mathcal{T}_{1: N}^{*}$ and estimation rules $\mathcal{E}_{1: N}^{*}$ are person-by-person optimal for (2) if the following relations hold for every $x_{0}$ in $\mathbb{R}^{2} \times[0,2 \pi)$ :

$$
\begin{aligned}
\mathcal{J}\left(x_{0}, \mathcal{T}_{1: N}^{*}, \mathcal{E}_{1: N}^{*}\right) & =\min _{\mathcal{T}_{1: N}} \mathcal{J}\left(x_{0}, \mathcal{T}_{1: N}, \mathcal{E}_{1: N}^{*}\right) \\
& =\min _{\mathcal{E}_{1: N}} \mathcal{J}\left(x_{0}, \mathcal{T}_{1: N}^{*}, \mathcal{E}_{1: N}\right)
\end{aligned}
$$

Equation (5) implies that with the transmission policies $\mathcal{T}_{1: N}^{*}$ fixed, the estimation rules $\mathcal{E}_{1: N}^{*}$ minimize the cost functional (2), and vice versa.

We maintain the following assumption throughout the paper.

Assumption II.4: Let $\mathfrak{B}$ be a Borel $\sigma$-algebra on $\mathbb{R}^{2} \times[0,2 \pi)$. We assume that $\mathbf{v}_{k}$ and $\phi_{k}$ in (1) are random processes for which the following hold for every $k$ in $\{1, \cdots, N\}$ :

1) For every Borel set $\mathbb{A}$ in $\mathfrak{B}$, the function $x \mapsto \mathbb{P}\left(\mathbf{x}_{k} \in \mathbb{A} \mid \mathbf{x}_{k-1}=x\right)$ is well-defined and continuous.

2) For every non-empty open set $\mathbb{O}$, the function $x \mapsto \mathbb{P}\left(\mathbf{x}_{k} \in \mathbb{O} \mid \mathbf{x}_{k-1}=x\right)$ is positive for all $x$ in $\mathbb{R}^{2} \times[0,2 \pi)$.

\footnotetext{
${ }^{3}$ We do not lose any optimality from imposing these structures. This can be verified by similar arguments as in Lemma 1 and Lemma 3 of

${ }^{4}$ See Appendix $\mathrm{B}$ for a detailed description of randomized transmission policies policies.

${ }^{5}$ The underlying SPP model and the initial condition $\mathbf{x}_{0}=x_{0}$ are common knowledge to both the sensing unit and estimator.
} [2]. 
To find a solution to Problem II.1, we decompose the problem into a set of $N$ sub-problems, which we solve sequentially. We start by describing the so-called Two-Player Optimal Stopping Problem, which we use to decompose Problem II.1 into sub-problems. We then describe how to obtain a solution to Problem II.1 by solving the subproblems.

Problem II.5 (Two-Player Optimal Stopping Problem): Given positive real numbers $\left\{c_{j}^{\prime}\right\}_{j=k}^{N}$, find policies $\mathcal{T}_{k: N}^{<k-1>}$ and rules $\mathcal{E}_{k: N}^{<k-1>}$ that are optimal for the following cost functional:

$$
\begin{aligned}
& \mathcal{J}_{k}\left(x_{k-1}, \mathcal{T}_{k: N}^{<k-1>}, \mathcal{E}_{k: N}^{<k-1>}\right) \\
& =\mathbb{E}\left[\sum_{j=k}^{\mathbf{K}} d^{2}\left(\mathbf{x}_{j}, \hat{\mathbf{x}}_{j}\right)+c_{\mathbf{K}}^{\prime} \cdot \mathbf{R}_{\mathbf{K}} \mid \mathbf{x}_{k-1}=x_{k-1}, \mathcal{T}_{k: N}^{<k-1>}, \mathcal{E}_{k: N}^{<k-1>}\right]
\end{aligned}
$$

subject to (1) with the initial condition $\mathbf{x}_{k-1}=x_{k-1}$ and

$$
\begin{aligned}
\mathbf{R}_{j} & =\mathcal{T}_{j}^{<k-1>}\left(x_{k-1}, \mathbf{x}_{j}\right) \\
\hat{\mathbf{x}}_{j} & = \begin{cases}\mathcal{E}_{j}^{<k-1>}\left(x_{k-1}\right) & \text { if } \mathbf{R}_{j}=0 \\
\mathbf{x}_{j} & \text { otherwise }\end{cases}
\end{aligned}
$$

for each $j$ in $\{k, \cdots, N\}$, where

$$
\mathbf{K}=\min \left\{k \leq j \leq N \mid \mathbf{R}_{j}=1\right\}
$$

Note that the total expected cost (6) consists of running costs $d^{2}\left(\mathbf{x}_{j}, \hat{\mathbf{x}}_{j}\right)$ and stopping costs $c_{j}^{\prime}$.

Similar to Definitions II.2 and II.3, we adopt two notions of optimality for Problem II.5 as follows.

Definition II.6: We say that policies $\mathcal{T}_{k: N}^{*<k-1>}$ and rules $\mathcal{E}_{k: N}^{*<-1>}$ are jointly optimal for (6) if they achieve the global minimum for every $x_{k-1}$ in $\mathbb{R}^{2} \times[0,2 \pi)$.

Definition II.7: We say that policies $\mathcal{T}_{k: N}^{*<k-1>}$ and rules $\mathcal{E}_{k: N}^{*<k-1>}$ are person-by-person optimal for (6) if the following relations hold for every $x_{k-1}$ in $\mathbb{R}^{2} \times[0,2 \pi)$ :

$$
\begin{aligned}
\mathcal{J}_{k}\left(x_{k-1}, \mathcal{T}_{k: N}^{*<k-1>}, \mathcal{E}_{k: N}^{*<k-1>}\right) & =\min _{\mathcal{T}_{k: N}^{<k-1}} \mathcal{J}_{k}\left(x_{k-1}, \mathcal{T}_{k: N}^{<k-1>}, \mathcal{E}_{k: N}^{*<k-1>}\right) \\
& =\min _{\mathcal{E}_{k: N-1>}^{<k}} \mathcal{J}_{k}\left(x_{k-1}, \mathcal{T}_{k: N}^{*<k-1>}, \mathcal{E}_{k: N}^{<k-1>}\right)
\end{aligned}
$$

To explain how to decompose Problem II.1 into sub-problems, we consider recursive computations of constants $\left\{c_{j}^{\prime}\right\}_{j=k}^{N}$ described as follows: Suppose that policies $\mathcal{T}_{j+1: N}^{<j>}$ and rules $\mathcal{E}_{j+1: N}^{<j>}$ are given for all $j$ in $\{k, \cdots, N\}$. By proceeding backwards from $j=N$ to $j=k$, for each step $j$, let us compute

$$
c_{j}^{\prime}=c_{j}+\mathcal{J}_{j+1}\left(0, \mathcal{T}_{j+1: N}^{<j>}, \mathcal{E}_{j+1: N}^{<j>}\right)
$$

with $c_{N}^{\prime}=c_{N}$, where $c_{j}$ is given in 2 and $\mathcal{J}_{j+1}$ is defined in (6). In computing $\mathcal{J}_{j+1}\left(0, \mathcal{T}_{j+1: N}^{<j>}, \mathcal{E}_{j+1: N}^{<j>}\right)$, we use the constants $\left\{c_{l}^{\prime}\right\}_{l=j+1}^{N}$ that are obtained in preceding steps.

\footnotetext{
${ }^{6}$ We adopt a convention that $\mathbf{K}=N$ if $\mathbf{R}_{j}=0$ for all $j$ in $\{k, \cdots, N\}$.
} 
We describe the $k$-th sub-problem of Problem II.1 as follows.

Sub-problem $k$ : Given policies $\mathcal{T}_{j+1: N}^{<j>}$ and rules $\mathcal{E}_{j+1: N}^{<j>}$ for all $j$ in $\{k, \cdots, N\}$, let us compute constants $\left\{c_{j}^{\prime}\right\}_{j=k}^{N}$ according to 9 . Using $\left\{c_{j}^{\prime}\right\}_{j=k}^{N}$, find a solution $\mathcal{T}_{k: N}^{<k-1>}$ and $\mathcal{E}_{k: N}^{<k-1>}$ to Problem II.5.

Our main strategy for solving Problem II.1 is as follows: We solve each Sub-problem $k$ backwards in time from $k=N$ to $k=1$, where for each Sub-problem $k$ we use solutions to all preceding sub-problems, i.e., $\boldsymbol{T}_{j+1: N}^{<j>}$ and $\mathcal{E}_{j+1: N}^{<j>}$ for all $j$ in $\{k, \cdots, N\}$, to compute the constants $\left\{c_{j}^{\prime}\right\}_{j=k}^{N}$. Once solutions to all the sub-problems are found, we determine transmission policies $\mathcal{T}_{1: N}$ and estimation rules $\mathcal{E}_{1: N}$ for Problem II.1 in the following way:

$$
\begin{array}{r}
\mathcal{T}_{j}\left(k-1, x_{k-1}, x_{j}\right)=\mathcal{T}_{j}^{<k-1>}\left(x_{k-1}, x_{j}\right) \\
\mathcal{E}_{j}\left(k-1, x_{k-1}\right)=\mathcal{E}_{j}^{<k-1>}\left(x_{k-1}\right)
\end{array}
$$

for each $j$ in $\{k, \cdots, N\}$ and $k$ in $\{1, \cdots, N\}$. It can be verified that the transmission policies and estimation rules determined by (10) are a solution to Problem II.1.

\section{Brief Survey of Related Work}

Finite time-horizon problem formulations are considered in [2]-[5]. The authors of [3] found a jointly optimal solution for a remote estimation problem under first-order linear processes driven by Gaussian noise where it is shown that transmission policies of jointly optimal solutions are of threshold-type. An iterative procedure for finding transmission policies and estimation rules was proposed in [2]. The authors performed a convergence analysis on the proposed procedure in the same problem formulation of [3], which essentially leads to an alternative proof of the main results of [3]. The work of [4] considered a problem setting in which the sensing unit has an energy harvesting capability. Preliminary results of our work were presented in [5] under some technical assumptions ${ }^{7}$

Infinite time-horizon formulations are considered in [6]-[9]. The authors of [6] studied the structure of optimal transmission policies for a remote estimation problem under linear processes driven by Gaussian noise, and proposed a procedure based on the value iteration algorithm to compute an optimal policy. In [7], an algorithm for finding a sub-optimal solution was proposed. The authors showed that when the underlying process is linear and driven by Gaussian noise, the proposed algorithm incurs a cost that is within a constant factor of the optimum. While the question of whether transmission policies of jointly optimal solutions are of threshold-type for the problems under multi-dimensional linear processes remains unanswered, the authors of [8] analyzed the performance of thresholdtype transmission policies for such problems. In [9], the authors proposed a polynomial approximation-based method to find sub-optimal transmission policies.

\footnotetext{
${ }^{7}$ The analysis and results presented in this work can be readily extended to the problem formulation considered in [5]. Due to the space constraints, we will focus on SPP models.
} 
Our problem formulation and methods are distinguished from previous ones found in literature by the following facts:

1) We adopt a random process model that is nonlinear.

2) We do not impose any structural assumptions on transmission policies and estimation rules that result in the loss of optimality.

3) We investigate optimization of a given performance criterion over both transmission policies and estimation rules.

\section{Two-Player Optimal Stopping Problem}

In this section, we investigate Sub-problem $k$ in which, to determine the constants $\left\{c_{j}^{\prime}\right\}_{j=k}^{N}$, we use solutions to the preceding sub-problems - Sub-problem $N$ to Sub-problem $k+1$. We start by re-writing (6) into a suitable form using the following definition.

Definition III.1: For each $j$ in $\{k, \cdots, N\}$, we define a (random) function $\mathcal{P}_{j}: \mathbb{R}^{2} \times[0,2 \pi) \rightarrow\{0,1\}$ and a variable $\hat{x}_{j}$ in $\mathbb{R}^{2} \times[0,2 \pi)$ as follows:

$$
\begin{gathered}
\mathcal{P}_{j}\left(x_{j}\right)=\mathcal{T}_{j}^{<k-1>}\left(0, x_{j}\right) \\
\hat{x}_{j}=\mathcal{E}_{j}^{<k-1>}(0)
\end{gathered}
$$

We refer to $\mathcal{P}_{j}$ and $\hat{x}_{j}$ as the (randomized) policy and estimate at time $j$ (for the initial condition $\mathbf{x}_{k-1}=0$ ), respectively ${ }^{8}$

Given that $\mathbf{x}_{k-1}=0$, we can re-write (6) as follows 9

$$
\mathbb{E}_{\mathbf{x}_{k}}\left[J_{k}\left(\mathbf{x}_{k}, \mathcal{P}_{k: N}, \hat{x}_{k: N}\right)\right]
$$

subject to (1) with the initial condition $\mathbf{x}_{k-1}=0$ and

$$
\mathbf{R}_{j}=\mathcal{P}_{j}\left(\mathbf{x}_{j}\right)
$$

for each $j$ in $\{k, \cdots, N\}$, where $J_{k}$ is recursively defined as follows:

$$
\begin{aligned}
& J_{j}\left(x_{j}, \mathcal{P}_{j: N}, \hat{x}_{j: N}\right) \\
& =\left(d^{2}\left(x_{j}, \hat{x}_{j}\right)+\mathbb{E}_{\mathbf{x}_{j+1}}\left[J_{j+1}\left(\mathbf{x}_{j+1}, \boldsymbol{P}_{j+1: N}, \hat{x}_{j+1: N}\right) \mid \mathbf{x}_{j}=x_{j}\right]\right) \cdot\left(1-\mathbf{R}_{j}\right)+c_{j}^{\prime} \cdot \mathbf{R}_{j}
\end{aligned}
$$

for each $j$ in $\{k, \cdots, N\}$ with $J_{N+1}=0$. Note that $J_{j}$ satisfies the following for every $j$ in $\{k, \cdots, N\}$ :

$$
\begin{aligned}
& \mathbb{E}_{\mathbf{x}_{j}}\left[J_{j}\left(\mathbf{x}_{j}, \mathcal{P}_{j: N}, \hat{x}_{j: N}\right) \mid \mathbf{R}_{k}=0, \cdots, \mathbf{R}_{j-1}=0\right] \\
& =\left(\mathbb{E}_{\mathbf{x}_{j}}\left[d^{2}\left(\mathbf{x}_{j}, \hat{x}_{j}\right) \mid \mathbf{R}_{k}=0, \cdots, \mathbf{R}_{j}=0\right]+\mathbb{E}_{\mathbf{x}_{j+1}}\left[J_{j+1}\left(\mathbf{x}_{j+1}, \boldsymbol{P}_{j+1: N}, \hat{x}_{j+1: N}\right) \mid \mathbf{R}_{k}=0, \cdots, \mathbf{R}_{j}=0\right]\right) \\
& \quad \cdot \mathbb{P}\left(\mathbf{R}_{j}=0 \mid \mathbf{R}_{k}=0, \cdots, \mathbf{R}_{j-1}=0\right)+c_{j}^{\prime} \cdot \mathbb{P}\left(\mathbf{R}_{j}=1 \mid \mathbf{R}_{k}=0, \cdots, \mathbf{R}_{j-1}=0\right)
\end{aligned}
$$

\footnotetext{
${ }^{8}$ See Appendix $\mathrm{B}$ for a detailed description of randomized policies.
}

${ }^{9}$ For concise presentation, we will omit the dependence of the cost functional 12] on the initial condition unless it is necessary. 
We will proceed with finding an optimal solution $\mathcal{P}_{k: N}^{*}$ and $\hat{x}_{k: N}^{*}$ for 12. Remark III.2 given below explains how we can derive a solution to Sub-problem $k$ from $\mathcal{P}_{k: N}^{*}$ and $\hat{x}_{k: N}^{*}$.

Remark III.2: Consider the transformations given below:

$$
\begin{aligned}
M\left(x_{k-1}, x_{j}\right) & =\left(\begin{array}{ccc}
\cos \theta_{k-1} & \sin \theta_{k-1} & 0 \\
-\sin \theta_{k-1} & \cos \theta_{k-1} & 0 \\
0 & 0 & 1
\end{array}\right) \cdot\left(\begin{array}{c}
p_{1, j}-p_{1, k-1} \\
p_{2, j}-p_{2, k-1} \\
\theta_{j}-\theta_{k-1}
\end{array}\right) \\
M^{\dagger}\left(x_{k-1}, x_{j}\right) & =\left(\begin{array}{ccc}
\cos \theta_{k-1} & -\sin \theta_{k-1} & 0 \\
\sin \theta_{k-1} & \cos \theta_{k-1} & 0 \\
0 & 0 & 1
\end{array}\right) \cdot\left(\begin{array}{c}
p_{1, j} \\
p_{2, j} \\
\theta_{j}
\end{array}\right)+\left(\begin{array}{c}
p_{1, k-1} \\
p_{2, k-1} \\
\theta_{k-1}
\end{array}\right)
\end{aligned}
$$

Suppose that $\mathcal{P}_{k: N}^{*}$ and $\hat{x}_{k: N}^{*}$ are optimal policies and estimates for (12), respectively, and that a solution to Subproblem $k$ are determined as follows: For each $j$ in $\{k, \cdots, N\}$,

$$
\begin{gathered}
\mathcal{T}_{j}^{*<k-1>}\left(x_{k-1}, x_{j}\right)=\mathcal{P}_{j}^{*}\left(M\left(x_{k-1}, x_{j}\right)\right) \\
\mathcal{E}_{j}^{*<k-1>}\left(x_{k-1}\right)=M^{\dagger}\left(x_{k-1}, \hat{x}_{j}^{*}\right)
\end{gathered}
$$

Based on Definition III.1, it can be verified that the following holds for all $x_{k-1}$ in $\mathbb{R}^{2} \times[0,2 \pi)$ :

$$
\mathcal{J}_{k}\left(x_{k-1}, \mathcal{T}_{k: N}^{*<k-1>}, \mathcal{E}_{k: N}^{*<k-1>}\right)=\mathbb{E}_{\mathbf{x}_{k}}\left[J_{k}\left(\mathbf{x}_{k}, \mathcal{P}_{k: N}^{*}, \hat{x}_{k: N}^{*}\right)\right]
$$

where $\mathcal{J}_{k}$ is defined in (6). This implies that the value of (6) evaluated at an optimal solution does not depend on the initial condition; and by finding an optimal solution for the sub-problem with the initial condition $\mathbf{x}_{k-1}=0$, we can derive a solution to Sub-problem $k$ using $[15$.

\section{A. Definitions and Preliminary Results}

We restate Definition $\amalg .6$ and Definition $\amalg .7$ as follows.

Definition III.3: We say that policies $\mathcal{P}_{k: N}^{*}$ and estimates $\hat{x}_{k: N}^{*}$ are jointly optimal for $[12$ if they achieve the global minimum.

Definition III.4: We say that policies $\mathcal{P}_{k: N}^{*}$ and estimates $\hat{x}_{k: N}^{*}$ are person-by-person optimal for (12) if the following relations hold:

$$
\begin{aligned}
\mathbb{E}_{\mathbf{x}_{k}}\left[J_{k}\left(\mathbf{x}_{k}, \mathcal{P}_{k: N}^{*}, \hat{x}_{k: N}^{*}\right)\right] & =\min _{\mathcal{P}_{k: N}} \mathbb{E}_{\mathbf{x}_{k}}\left[J_{k}\left(\mathbf{x}_{k}, \mathcal{P}_{k: N}, \hat{x}_{k: N}^{*}\right)\right] \\
& =\min _{\hat{x}_{k: N}} \mathbb{E}_{\mathbf{x}_{k}}\left[J_{k}\left(\mathbf{x}_{k}, \mathcal{P}_{k: N}^{*}, \hat{x}_{k: N}\right)\right]
\end{aligned}
$$

In what follows, we define best response mappings $\mathfrak{P}$ and $\mathfrak{X}$.

Definition III.5: Given estimates $\hat{x}_{k: N}$, we define $\mathfrak{P}\left(\hat{x}_{k: N}\right)$ as the collection of policies $\mathcal{P}_{k: N}$ satisfying

$$
\mathbb{E}_{\mathbf{x}_{k}}\left[J_{k}\left(\mathbf{x}_{k}, \mathcal{P}_{k: N}, \hat{x}_{k: N}\right)\right]=\min _{\mathcal{P}_{k: N}^{\prime}} \mathbb{E}_{\mathbf{x}_{k}}\left[J_{k}\left(\mathbf{x}_{k}, \mathcal{P}_{k: N}^{\prime}, \hat{x}_{k: N}\right)\right]
$$

Definition III.6: Given policies $\mathcal{P}_{k: N}$, we define $\mathfrak{X}\left(\mathcal{P}_{k: N}\right)$ as the collection of estimates $\hat{x}_{k: N}$ satisfying

$$
\mathbb{E}_{\mathbf{x}_{k}}\left[J_{k}\left(\mathbf{x}_{k}, \mathcal{P}_{k: N}, \hat{x}_{k: N}\right)\right]=\min _{\hat{x}_{k: N}^{\prime}} \mathbb{E}_{\mathbf{x}_{k}}\left[J_{k}\left(\mathbf{x}_{k}, \mathcal{P}_{k: N}, \hat{x}_{k: N}^{\prime}\right)\right]
$$


Definition III.7: Policies $\mathcal{P}_{k: N}$ are said to be degenerate if there exists $j_{0}$ in $\{k, \cdots, N\}$ for which it holds that

$$
\mathbb{P}\left(\mathbf{R}_{j_{0}}=0 \mid \mathbf{R}_{k}=0, \cdots, \mathbf{R}_{j_{0}-1}=0\right)=0
$$

Remark III.8: Let $\mathcal{P}_{k: N}$ be degenerate policies such that $\left[16\right.$ holds for $j_{0}$ in $\{k, \cdots, N\}$. From (14), we can derive that

$$
\mathbb{E}_{\mathbf{x}_{j_{0}}}\left[J_{j_{0}}\left(\mathbf{x}_{j_{0}}, \mathcal{P}_{j_{0}: N}, \hat{x}_{j_{0}: N}\right) \mid \mathbf{R}_{k}=0, \cdots, \mathbf{R}_{j_{0}-1}=0\right]=c_{j_{0}}^{\prime}
$$

from which we can infer that the cost 12$]$ does not depend on the choice of estimates $\hat{x}_{j_{0}: N}$.

Proposition III.9: Suppose that non-degenerate policies $\mathcal{P}_{k: N}$ and estimates $\hat{x}_{k: N}$ are given. The policies $\mathcal{P}_{k: N}$ belong to $\mathfrak{P}\left(\hat{x}_{k: N}\right)$ if and only if the following holds for all $j$ in $\{k, \cdots, N\}$ :

$$
\begin{aligned}
& \mathbb{E}_{\mathbf{x}_{j}}\left[J_{j}\left(\mathbf{x}_{j}, \mathcal{P}_{j: N}, \hat{x}_{j: N}\right) \mid \mathbf{R}_{k}=0, \cdots, \mathbf{R}_{j-1}=0\right] \\
& =\mathbb{E}_{\mathbf{x}_{j}}\left[J_{j}^{*}\left(\mathbf{x}_{j}, \hat{x}_{j: N}\right) \mid \mathbf{R}_{k}=0, \cdots, \mathbf{R}_{j-1}=0\right]
\end{aligned}
$$

where for each $j$ in $\{k, \cdots, N\}$,

$$
J_{j}^{*}\left(x_{j}, \hat{x}_{j: N}\right)=\min \left\{d^{2}\left(x_{j}, \hat{x}_{j}\right)+\mathbb{E}_{\mathbf{x}_{j+1}}\left[J_{j+1}^{*}\left(\mathbf{x}_{j+1}, \hat{x}_{j+1: N}\right) \mid \mathbf{x}_{j}=x_{j}\right], c_{j}^{\prime}\right\}
$$

with $J_{N+1}^{*}=0$

The proof follows from (14), Definition III.5, and the fact that

$$
\min _{\mathcal{P}_{k: N}^{\prime}} \mathbb{E}_{\mathbf{x}_{k}}\left[J_{k}\left(\mathbf{x}_{k}, \mathcal{P}_{k: N}^{\prime}, \hat{x}_{k: N}\right)\right]=\mathbb{E}_{\mathbf{x}_{k}}\left[J_{k}^{*}\left(\mathbf{x}_{k}, \hat{x}_{k: N}\right)\right]
$$

We omit the detail for brevity.

Corollary III.10: Given estimates $\hat{x}_{k: N}$, for each $j$ in $\{k, \cdots, N\}$, let us define sets $\overline{\mathbb{D}}_{j}$ and $\underline{\mathbb{D}}_{j}$ as follows:

$$
\begin{aligned}
& \overline{\mathbb{D}}_{j}=\left\{x_{j} \in \mathbb{R}^{2} \times[0,2 \pi) \mid d^{2}\left(x_{j}, \hat{x}_{j}\right)+\mathbb{E}_{\mathbf{x}_{j+1}}\left[J_{j+1}^{*}\left(\mathbf{x}_{j+1}, \hat{x}_{j+1: N}\right) \mid \mathbf{x}_{j}=x_{j}\right] \leq c_{j}^{\prime}\right\} \\
& \underline{\mathbb{D}}_{j}=\left\{x_{j} \in \mathbb{R}^{2} \times[0,2 \pi) \mid d^{2}\left(x_{j}, \hat{x}_{j}\right)+\mathbb{E}_{\mathbf{x}_{j+1}}\left[J_{j+1}^{*}\left(\mathbf{x}_{j+1}, \hat{x}_{j+1: N}\right) \mid \mathbf{x}_{j}=x_{j}\right]<c_{j}^{\prime}\right\}
\end{aligned}
$$

Consider (deterministic) policies $\mathcal{P}_{k: N}$ defined by

$$
\mathcal{P}_{j}\left(x_{j}\right)= \begin{cases}0 & \text { if } x_{j} \in \mathbb{D}_{j} \\ 1 & \text { otherwise }\end{cases}
$$

for each $j$ in $\{k, \cdots, N\}$, where $\mathbb{D}_{j}$ is a measurable set satisfying $\underline{\mathbb{D}}_{j} \subseteq \mathbb{D}_{j} \subseteq \overline{\mathbb{D}}_{j}$. The policies $\mathcal{P}_{k: N}$ belong to $\mathfrak{P}\left(\hat{x}_{k: N}\right)$.

Proposition III.11: Consider that non-degenerate policies $\mathcal{P}_{k: N}$ and estimates $\hat{x}_{k: N}$ are given. The estimates $\hat{x}_{k: N}$ belong to $\mathfrak{X}\left(\mathcal{P}_{k: N}\right)$ if and only if the following holds for all $j$ in $\{k, \cdots, N\}$ :

$$
\begin{aligned}
& \mathbb{E}_{\mathbf{x}_{j}}\left[d^{2}\left(\mathbf{x}_{j}, \hat{x}_{j}\right) \mid \mathbf{R}_{k}=0, \cdots, \mathbf{R}_{j}=0\right] \\
& =\min _{\hat{x}_{j}^{\prime} \in \mathbb{R}^{2} \times[0,2 \pi)} \mathbb{E}_{\mathbf{x}_{j}}\left[d^{2}\left(\mathbf{x}_{j}, \hat{x}_{j}^{\prime}\right) \mid \mathbf{R}_{k}=0, \cdots, \mathbf{R}_{j}=0\right]
\end{aligned}
$$

The proof follows from (14) and Definition III.6. We omit the detail for brevity. 
Corollary III.12: Given non-degenerate policies $\mathcal{P}_{k: N}$, for each $j$ in $\{k, \cdots, N\}$, let us consider an estimate $\hat{x}_{j}=\left(\begin{array}{lll}\hat{p}_{1, j} & \hat{p}_{2, j} & \hat{\theta}_{j}\end{array}\right)^{T}$ determined as follows:

$$
\begin{aligned}
& \hat{p}_{1, j}=\mathbb{E}\left[\mathbf{p}_{1, j} \mid \mathbf{R}_{k}=0, \cdots, \mathbf{R}_{j}=0\right] \\
& \hat{p}_{2, j}=\mathbb{E}\left[\mathbf{p}_{2, j} \mid \mathbf{R}_{k}=0, \cdots, \mathbf{R}_{j}=0\right]
\end{aligned}
$$

and $\hat{\theta}_{j}$ takes a value in $[0,2 \pi)$ that satisfies

$$
\begin{aligned}
\sin \hat{\theta}_{j} & =\alpha^{-1} \cdot \mathbb{E}\left[\sin \boldsymbol{\theta}_{j} \mid \mathbf{R}_{k}=0, \cdots, \mathbf{R}_{j}=0\right] \\
\cos \hat{\theta}_{j} & =\alpha^{-1} \cdot \mathbb{E}\left[\cos \boldsymbol{\theta}_{j} \mid \mathbf{R}_{k}=0, \cdots, \mathbf{R}_{j}=0\right]
\end{aligned}
$$

provided

$$
\alpha=\mathbb{E}^{2}\left[\sin \boldsymbol{\theta}_{j} \mid \mathbf{R}_{k}=0, \cdots, \mathbf{R}_{j}=0\right]+\mathbb{E}^{2}\left[\cos \boldsymbol{\theta}_{j} \mid \mathbf{R}_{k}=0, \cdots, \mathbf{R}_{j}=0\right]
$$

is non-zero; otherwise $\hat{\theta}_{j}$ takes any value in $[0,2 \pi)$. The estimates $\hat{x}_{k: N}$ belong to $\mathfrak{X}\left(\mathcal{P}_{k: N}\right)$.

Proposition III.13: Consider functions $\left\{\mathcal{G}_{j}\right\}_{j=k}^{N}$ defined as follows ${ }^{10}$ For each $j$ in $\{k, \cdots, N\}$,

$$
\mathcal{G}_{j}\left(x_{j-1}, \hat{x}_{j: N}\right) \stackrel{\text { def }}{=} \mathbb{E}_{\mathbf{x}_{j}}\left[J_{j}^{*}\left(\mathbf{x}_{j}, \hat{x}_{j: N}\right) \mid \mathbf{x}_{j-1}=x_{j-1}\right]
$$

where $J_{j}^{*}$ is given in $(18)$. The functions $\left\{\mathcal{G}_{j}\right\}_{j=k}^{N}$ are all continuous.

The proof is given in Appendix E

\section{B. Existence of a Jointly Optimal Solution}

Proposition III.14: Let policies $\mathcal{P}_{k: N}^{*}$ and estimates $\hat{x}_{k: N}^{*}$ are jointly optimal for [12). The policies $\mathcal{P}_{k: N}^{*}$ are not degenerate in the sense of Definition III.7.

The proof is given in Appendix $\mathrm{F}$

Theorem III.15: There exist policies $\mathcal{P}_{k: N}^{*}$ and estimates $\hat{x}_{k: N}^{*}$ that are jointly optimal for (12).

To prove Theorem III.15, we need the following lemma.

Lemma III.16: Let us define

$$
\mathcal{G}\left(\hat{x}_{k: N}\right) \stackrel{\text { def }}{=} \mathbb{E}_{\mathbf{x}_{k}}\left[J_{k}^{*}\left(\mathbf{x}_{k}, \hat{x}_{k: N}\right)\right]
$$

with the initial condition $\mathbf{x}_{k-1}=0$, where $J_{k}^{*}$ is defined in $(18)$. There exists a compact set $\mathbb{K} \subset\left(\mathbb{R}^{2} \times[0,2 \pi)\right)^{N-k+1}$ for which the following holds for all $\hat{x}_{k: N}$ in $\left(\mathbb{R}^{2} \times[0,2 \pi)\right)^{N-k+1}$ :

The proof is given in Appendix $\mathrm{F}$

$$
\inf _{\hat{x}_{k: N}^{\prime} \in \mathbb{K}} \mathcal{G}\left(\hat{x}_{k: N}^{\prime}\right) \leq \mathcal{G}\left(\hat{x}_{k: N}\right)
$$

Proof of Theorem III.15. Recall the definitions of $\mathcal{G}_{k}$ and $\mathcal{G}$ given in 23) and (24), respectively. According to Proposition III.13 and by the fact that $\mathcal{G}\left(\hat{x}_{k: N}\right)=\mathcal{G}_{k}\left(0, \hat{x}_{k: N}\right)$, we can see that $\mathcal{G}$ is a continuous function. Note

${ }^{10}$ Note that $\mathcal{G}_{j}$ is a function defined on $\left(\mathbb{R}^{2} \times[0,2 \pi)\right)^{N-j+2}$. See Appendix A for some remarks on the continuity of functions on a product space. 
that Lemma III.16 implies that, if it exists, a global minimizer of $\mathcal{G}$ resides in a compact set. In what regards to finding a global minimizer, without loss of generality, we may assume that the domain of $\mathcal{G}$ is compact. Hence, by the continuity of $\mathcal{G}$ and compactness of its domain, there exist estimates $\hat{x}_{k: N}^{*}$ that achieve the global minimum of $\mathcal{G}$.

Next, let us choose policies $\mathcal{P}_{k: N}^{*}$ belonging to $\mathfrak{P}\left(\hat{x}_{k: N}^{*}\right)$ using, for instance, Corollary III.10 By the definition of $\mathcal{G}$ given as in 24 and by the fact that $\hat{x}_{k: N}^{*}$ is a global minimizer of $\mathcal{G}$, we conclude that the policies $\mathcal{P}_{k: N}^{*}$ and the estimates $x_{k: N}^{*}$ are jointly optimal for $[12]$.

\section{Iterative Procedure for Finding a Person-by-Person Optimal Solution}

As numerically illustrated in [2], the function $\mathcal{G}$ in 24] may be non-convex; consequently, finding a jointly optimal solution for (12) would be computationally intractable. Instead, we seek a person-by-person optimal solution based on Procedure 1 described below. In the procedure, $\eta$ is a pre-selected non-negative constant that determines a stopping criterion (Line 17), and the function $\mathcal{G}$ is defined in 24].

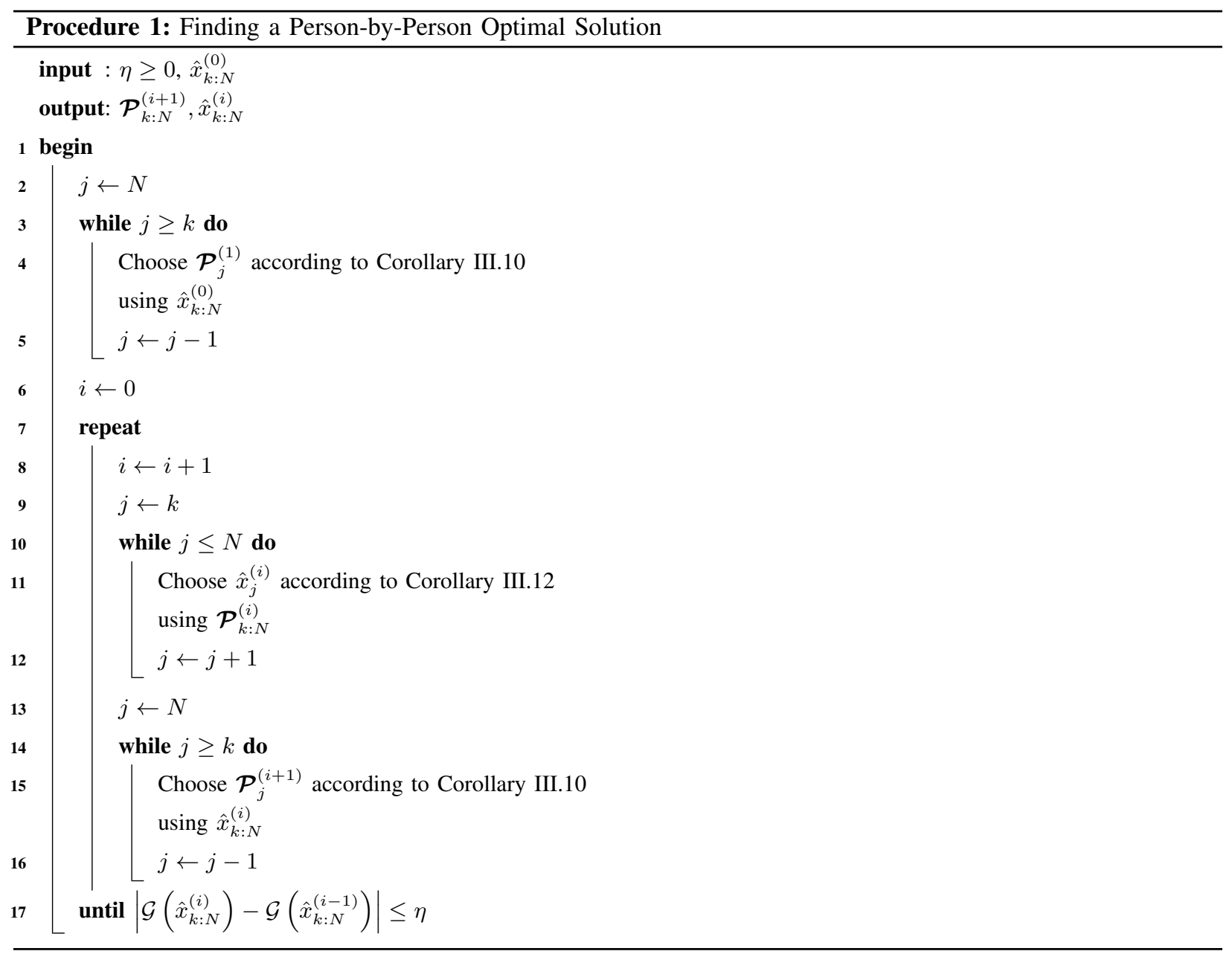


Let $\left\{\left(\mathcal{P}_{k: N}^{(i)}, \hat{x}_{k: N}^{(i)}\right)\right\}_{i \in \mathbb{N}}$ be a sequence of solutions generated through repeated computations of policies and estimates by Procedure 1 (Line $2-16$ ). In the rest of this section, we discuss convergence of the sequence to a person-by-person optimal solution. We first define convergence of policies and estimates. For notational convenience, we adopt the following: Let $\mathfrak{B}$ be a Borel $\sigma$-algebra on $\mathbb{R}^{2} \times[0,2 \pi)$. Given $\left\{\mathcal{P}_{k: N}^{(i)}\right\}_{i \in \mathbb{N}}$ and $\mathcal{P}_{k: N}$, let us define the following: For each $\mathbb{A}$ in $\mathfrak{B}$,

$$
\begin{aligned}
& \mu_{j \mid j}^{(i)}(\mathbb{A})=\mathbb{P}\left(\mathbf{x}_{j} \in \mathbb{A} \mid \mathbf{R}_{k}^{(i)}=0, \cdots, \mathbf{R}_{j}^{(i)}=0\right) \\
& \mu_{j \mid j}(\mathbb{A})=\mathbb{P}\left(\mathbf{x}_{j} \in \mathbb{A} \mid \mathbf{R}_{k}=0, \cdots, \mathbf{R}_{j}=0\right)
\end{aligned}
$$

subject to

$$
\begin{aligned}
\mathbf{R}_{j}^{(i)} & =\mathcal{P}_{j}^{(i)}\left(\mathbf{x}_{j}\right) \\
\mathbf{R}_{j} & =\mathcal{P}_{j}\left(\mathbf{x}_{j}\right)
\end{aligned}
$$

for all $i$ in $\mathbb{N}$ and $j$ in $\{k, \cdots, N\}$.

Definition III.17: Let $\left\{\mathcal{P}_{k: N}^{(i)}\right\}_{i \in \mathbb{N}}$ be a sequence of policies. We say that the sequence converges to $\mathcal{P}_{k: N}$ if the following hold for all $j$ in $\{k, \cdots, N\}$ :

$$
\mu_{j \mid j}^{(i)} \rightarrow \mu_{j \mid j}
$$

and

$$
\mathbb{P}\left(\mathbf{R}_{j}^{(i)}=0 \mid \mathbf{R}_{k}^{(i)}=0, \cdots, \mathbf{R}_{j-1}^{(i)}=0\right) \rightarrow \mathbb{P}\left(\mathbf{R}_{j}=0 \mid \mathbf{R}_{k}=0, \cdots, \mathbf{R}_{j-1}=0\right)
$$

subject to 26] ${ }^{11}$ We denote the convergence by $\mathcal{P}_{k: N}^{(i)} \Rightarrow \mathcal{P}_{k: N}$. In addition, we say that two sets of policies $\mathcal{P}_{k: N}$ and $\mathcal{P}_{k: N}^{\prime}$ are equal if the following hold for all $j$ in $\{k, \cdots, N\}$ :

$$
\mu_{j \mid j}=\mu_{j \mid j}^{\prime}
$$

and

$$
\mathbb{P}\left(\mathbf{R}_{j}=0 \mid \mathbf{R}_{k}=0, \cdots, \mathbf{R}_{j-1}=0\right)=\mathbb{P}\left(\mathbf{R}_{j}^{\prime}=0 \mid \mathbf{R}_{k}^{\prime}=0, \cdots, \mathbf{R}_{j-1}^{\prime}=0\right)
$$

subject to $\mathbf{R}_{j}=\mathcal{P}_{j}\left(\mathbf{x}_{j}\right)$ and $\mathbf{R}_{j}^{\prime}=\mathcal{P}_{j}^{\prime}\left(\mathbf{x}_{j}\right)$ for all $j$ in $\{k, \cdots, N\}$.

Remark III.18 (Uniqueness of the Limit of Policies): Suppose that a sequence of policies $\left\{\mathcal{P}_{k: N}^{(i)}\right\}_{i \in \mathbb{N}}$ converges to both $\mathcal{P}_{k: N}$ and $\mathcal{P}_{k: N}^{\prime}$. Then the two sets of the policies $\mathcal{P}_{k: N}$ and $\mathcal{P}_{k: N}^{\prime}$ are equal. To see this, using the definition of convergence of probability measures, we can derive that

$$
\int_{\mathbb{R}^{2} \times[0,2 \pi)} g \mathrm{~d} \mu_{j \mid j}=\int_{\mathbb{R}^{2} \times[0,2 \pi)} g \mathrm{~d} \mu_{j \mid j}^{\prime}
$$

for every bounded, continuous function $g: \mathbb{R}^{2} \times[0,2 \pi) \rightarrow \mathbb{R}$. Based on Lemma 9.3.2 in [10], we can see that 28] holds for all $j$ in $\{k, \cdots, N\}$.

\footnotetext{
${ }^{11}$ Equation 27a implies that the sequence of the probability measures $\left\{\mu_{j \mid j}^{(i)}\right\}_{i \in \mathbb{N}}$ converges to the probability measure $\mu_{j \mid j}$. See Chapter 9.3 of [10] for the definition of convergence of probability measures.
} 
Definition III.19: Let $\left\{\hat{x}_{k: N}^{(i)}\right\}_{i \in \mathbb{N}}$ be a sequence of estimates. We say that the sequence converges to $\hat{x}_{k: N}$ if the following holds for all $j$ in $\{k, \cdots, N\}$ :

$$
\lim _{i \rightarrow \infty} d\left(\hat{x}_{j}^{(i)}, \hat{x}_{j}\right)=0
$$

We denote the convergence by $\hat{x}_{k: N}^{(i)} \Rightarrow \hat{x}_{k: N}$. In addition, we say that two sets of estimates $\hat{x}_{k: N}$ and $\hat{x}_{k: N}^{\prime}$ are equal if the following holds for all $j$ in $\{k, \cdots, N\}$ :

$$
d\left(\hat{x}_{j}, \hat{x}_{j}^{\prime}\right)=0
$$

Definition III.20: Let $\left\{\mathcal{P}_{k: N}^{(i)}\right\}_{i \in \mathbb{N}}$ be a sequence of policies. We say that the policies are strictly non-degenerate if there exists a positive constant $\epsilon$ for which the following holds for all $i$ in $\mathbb{N}$ and $j$ in $\{k, \cdots, N\}$ :

$$
\mathbb{P}\left(\mathbf{R}_{j}^{(i)}=0 \mid \mathbf{R}_{k}^{(i)}=0, \cdots, \mathbf{R}_{j-1}^{(i)}=0\right) \geq \epsilon
$$

subject to 26a.

Recall that the sequence of solutions $\left\{\left(\mathcal{P}_{k: N}^{(i)}, \hat{x}_{k: N}^{(i)}\right)\right\}_{i \in \mathbb{N}}$ generated by Procedure 1 satisfies the following for all $i$ in $\mathbb{N}$ :

$$
\begin{gathered}
\mathcal{P}_{k: N}^{(i)} \in \mathfrak{P}\left(\hat{x}_{k: N}^{(i-1)}\right) \\
\hat{x}_{k: N}^{(i)} \in \mathfrak{X}\left(\mathcal{P}_{k: N}^{(i)}\right)
\end{gathered}
$$

The following theorem states convergence of the sequence to a person-by-person optimal solution.

Theorem III.21: Consider a sequence of solutions $\left\{\left(\mathcal{P}_{k: N}^{(i)}, \hat{x}_{k: N}^{(i)}\right)\right\}_{i \in \mathbb{N}}$ satisfying (33). Suppose that the policies $\left\{\mathcal{P}_{k: N}^{(i)}\right\}_{i \in \mathbb{N}}$ are strictly non-degenerate. Then, the sequence has a convergent subsequence, and the limit of any convergent subsequence is a person-by-person optimal solution.

To prove Theorem III.21, we need the following three lemmas.

Lemma III.22: Consider a sequence of solutions $\left\{\left(\mathcal{P}_{k: N}^{(i)}, \hat{x}_{k: N}^{(i)}\right)\right\}_{i \in \mathbb{N}}$ satisfying (33). Suppose that the policies $\left\{\mathcal{P}_{k: N}^{(i)}\right\}_{i \in \mathbb{N}}$ are strictly non-degenerate. Then the sequence $\left\{\hat{x}_{j}^{(i)}\right\}_{i \in \mathbb{N}}$ is bounded for all $j$ in $\{k, \cdots, N\}$.

Lemma III.22 is a special case of Lemma F.15 given in Appendix F.

Lemma III.23: Consider a sequence of solutions $\left\{\left(\mathcal{P}_{k: N}^{(i)}, \hat{x}_{k: N}^{(i)}\right)\right\}_{i \in \mathbb{N}}$ satisfying (33). Suppose that for an infinite subset $\left\{i_{l}\right\}_{l \in \mathbb{N}}$ of $\mathbb{N}$, the following hold: $\mathcal{P}_{k: N}^{\left(i_{l}\right)} \Rightarrow \mathcal{P}_{k: N}, \hat{x}_{k: N}^{\left(i_{l}\right)} \Rightarrow \hat{x}_{k: N}$, and $\hat{x}_{k: N}^{\left(i_{l}-1\right)} \Rightarrow \hat{x}_{k: N}^{\prime}$. Then the estimates $\hat{x}_{k: N}$ belong to $\mathfrak{X}\left(\mathcal{P}_{k: N}\right)$.

Lemma III.24: Consider a sequence of solutions $\left\{\left(\mathcal{P}_{k: N}^{(i)}, \hat{x}_{k: N}^{(i)}\right)\right\}_{i \in \mathbb{N}}$ satisfying (33). Suppose that the policies $\left\{\mathcal{P}_{k: N}^{(i)}\right\}_{i \in \mathbb{N}}$ are strictly non-degenerate and that for an infinite subset $\left\{i_{l}\right\}_{l \in \mathbb{N}}$ of $\mathbb{N}$, it holds that $\hat{x}_{k: N}^{\left(i_{l}-1\right)} \Rightarrow \hat{x}_{k: N}^{\prime}$ Then, the sequence $\left\{\mathcal{P}_{k: N}^{\left(i_{l}\right)}\right\}_{l \in \mathbb{N}}$ has a convergent subsequence, and the limit $\mathcal{P}_{k: N}$ of any convergent subsequence belongs to $\mathfrak{P}\left(\hat{x}_{k: N}^{\prime}\right)$.

The proofs of Lemmas III.23 and III.24 are given in Appendix G.

Proof of Theorem III.21. We first note that according to Lemma III.22 the sequence $\left\{\hat{x}_{j}^{(i)}\right\}_{i \in \mathbb{N}}$ is contained in a 
compact set for every $j$ in $\{k, \cdots, N\}{ }^{12}$ Hence, by the compactness, there exists an infinite subset $\mathbb{I}$ of $\mathbb{N}$ for which the subsequences $\left\{\hat{x}_{k: N}^{(i)}\right\}_{i \in \mathbb{I}}$ and $\left\{\hat{x}_{k: N}^{(i-1)}\right\}_{i \in \mathbb{I}}$ are both convergent. Let $\hat{x}_{k: N}$ and $\hat{x}_{k: N}^{\prime}$ be the respective limits of the subsequences. Also, according to Lemma III.24, there is an infinite subset $\mathbb{I}^{\prime}$ of $\mathbb{I}$ for which the subsequence $\left\{\mathcal{P}_{k: N}^{(i)}\right\}_{i \in \mathbb{I}^{\prime}}$ is convergent. Let $\mathcal{P}_{k: N}$ be the limit of this subsequence.

To complete the proof, it remains to show that $\mathcal{P}_{k: N}$ and $\hat{x}_{k: N}$ constitute a person-by-person optimal solution, i.e., it holds that

$$
\begin{gathered}
\mathcal{P}_{k: N} \in \mathfrak{P}\left(\hat{x}_{k: N}\right) \\
\hat{x}_{k: N} \in \mathfrak{X}\left(\mathcal{P}_{k: N}\right)
\end{gathered}
$$

Equation (34b) is ensured by Lemma III.23, hence it remains to show that 34a is true.

By contradiction, suppose that the policies $\mathcal{P}_{k: N}$ do not belong to $\mathfrak{P}\left(\hat{x}_{k: N}\right)$. Note that by Lemma III.24, $\mathcal{P}_{k: N}$ belong to $\mathfrak{P}\left(\hat{x}_{k: N}^{\prime}\right)$. We can see that the following relations hold for any policies $\mathcal{P}_{k: N}^{\prime}$ belonging to $\mathfrak{P}\left(\hat{x}_{k: N}\right)$ :

$$
\begin{aligned}
\mathcal{G}\left(\hat{x}_{k: N}\right) & =\mathbb{E}_{\mathbf{x}_{k}}\left[J_{k}\left(\mathbf{x}_{k}, \mathcal{P}_{k: N}^{\prime}, \hat{x}_{k: N}\right)\right] \\
& \stackrel{(\mathbf{i})}{<} \mathbb{E}_{\mathbf{x}_{k}}\left[J_{k}\left(\mathbf{x}_{k}, \mathcal{P}_{k: N}, \hat{x}_{k: N}\right)\right] \\
& \stackrel{(i \mathbf{i})}{\leq} \mathbb{E}_{\mathbf{x}_{k}}\left[J_{k}\left(\mathbf{x}_{k}, \mathcal{P}_{k: N}, \hat{x}_{k: N}^{\prime}\right)\right]=\mathcal{G}\left(\hat{x}_{k: N}^{\prime}\right)
\end{aligned}
$$

(i) follows from the hypothesis that $\mathcal{P}_{k: N} \notin \mathfrak{P}\left(\hat{x}_{k: N}\right)$; and (ii) is due to 34b. On the other hand, since $\mathcal{G}$ is non-negative and decreasing along the sequence $\left\{\hat{x}_{k: N}^{(i)}\right\}_{i \in \mathbb{N}}$, i.e., $\mathcal{G}\left(\hat{x}_{k: N}^{(i+1)}\right) \leq \mathcal{G}\left(\hat{x}_{k: N}^{(i)}\right)$ holds for all $i$ in $\mathbb{N}$, it holds that $\lim _{i \rightarrow \infty} \mathcal{G}\left(\hat{x}_{k: N}^{(i)}\right)=\alpha$ for some real number $\alpha$. In conjunction with the continuity of $\mathcal{G}$ (see Proposition III.13, this implies that $\mathcal{G}\left(\hat{x}_{k: N}\right)=\mathcal{G}\left(\hat{x}_{k: N}^{\prime}\right)=\alpha$ which contradicts 35]. Therefore we conclude that the policies $\mathcal{P}_{k: N}$ belong to $\mathfrak{P}\left(\hat{x}_{k: N}\right)$.

\section{Application to Tracking of Animal Movements}

In this section, we apply our results to estimation of animal movements over a costly communication link where the performance of the optimal scheme are illustrated using GPS data collected from a monitoring device mounted on an African buffalo 13 Fig. 2 shows the GPS track of the buffalo. To represent the movement of the buffalo, as described in [11], we adopt the SPP model (1) in which $\mathbf{v}_{k}$ and $\phi_{k}$ are the Weibull and Wrapped Cauchy random processes, respectively. Note that the probability density functions of $\mathbf{v}_{k}$ and $\phi_{k}$ are given as follows:

$$
\begin{aligned}
& f_{\mathbf{v}_{k}}(v)=\frac{a_{\mathbf{v}}}{s_{\mathbf{v}}}\left(\frac{v}{s_{\mathbf{v}}}\right)^{a_{\mathbf{v}}-1} e^{-\left(\frac{v}{s_{\mathbf{v}}}\right)^{a_{\mathbf{v}}}}, \text { for } v \geq 0 \\
& f_{\boldsymbol{\phi}_{k}}(\phi)=\frac{1}{2 \pi} \cdot \frac{1-a_{\boldsymbol{\phi}}^{2}}{1+a_{\boldsymbol{\phi}}^{2}-2 a_{\phi} \cos \left(\phi-m_{\boldsymbol{\phi}}\right)}
\end{aligned}
$$

\footnotetext{
${ }^{12}$ The metric space $\left(\mathbb{R}^{2} \times[0,2 \pi), d\right)$ is proper; hence for any bounded subset of $\mathbb{R}^{2} \times[0,2 \pi)$, we can find a compact set that contains the subset.

${ }^{13}$ The development and deployment of animal-borne monitoring devices were performed under a research grant NSF ECCS 1135726 . The GPS data were collected at the Gorongosa National Park, Mozambique.
} 


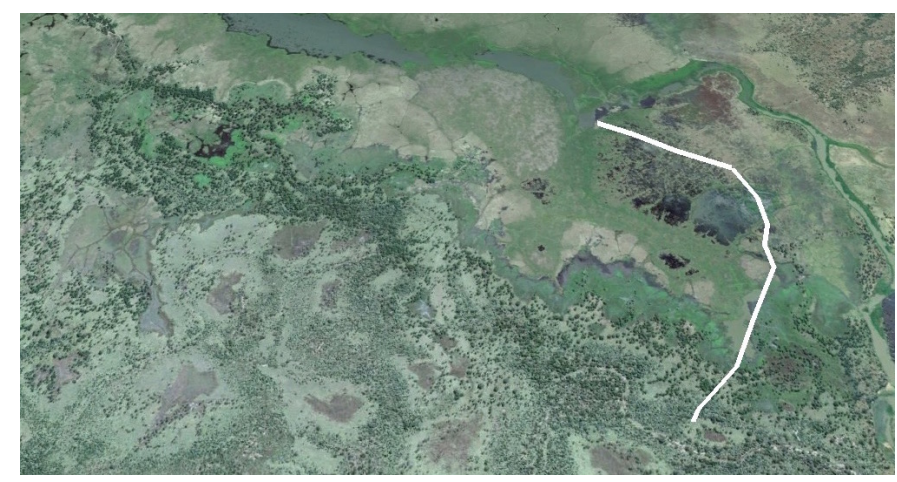

Fig. 2. A screenshot of a GPS track (the white trajectory) of an African buffalo in the Google Earth.

Using the collected GPS data, we compute the maximum likelihood estimates of the parameters for $(36)$ as follows:

$$
\begin{aligned}
\left(a_{\mathbf{v}}, s_{\mathbf{v}}\right) & =(1.35,4.66) \\
\left(a_{\boldsymbol{\phi}}, m_{\boldsymbol{\phi}}\right) & =(0.65,0.00)
\end{aligned}
$$

The graphs in Fig. 3 show comparisons between the resulting probability density functions and the histograms obtained from the GPS data.

We have selected the communication costs $c_{k}=10$ for all $k$ in $\{1, \cdots, N\}$ and the length of the time-horizon $N=100$. Using Procedure 1) (10), and [15), we have found the optimal remote estimation scheme where Fig. 4 illustrates the performance of the scheme in terms of the state estimation distortion computed by the metric $d\left(x_{k}, \hat{x}_{k}\right)$. Note that the (red) circles on the time axis ( $x$-axis) represents the time steps at which the sensing unit transmitted information on the full state $x_{k}$ to the estimator, and the state estimate $\hat{x}_{k}$ was set to $\hat{x}_{k}=x_{k}$ (hence $\left.d\left(x_{k}, \hat{x}_{k}\right)=0\right)$. Our experimental results show that the optimal scheme achieved the error of location estimation less than 5 meters compared to the total traveled distance of 372.53 meters; and the information transmissions occurred 32 times over 100 time steps. 

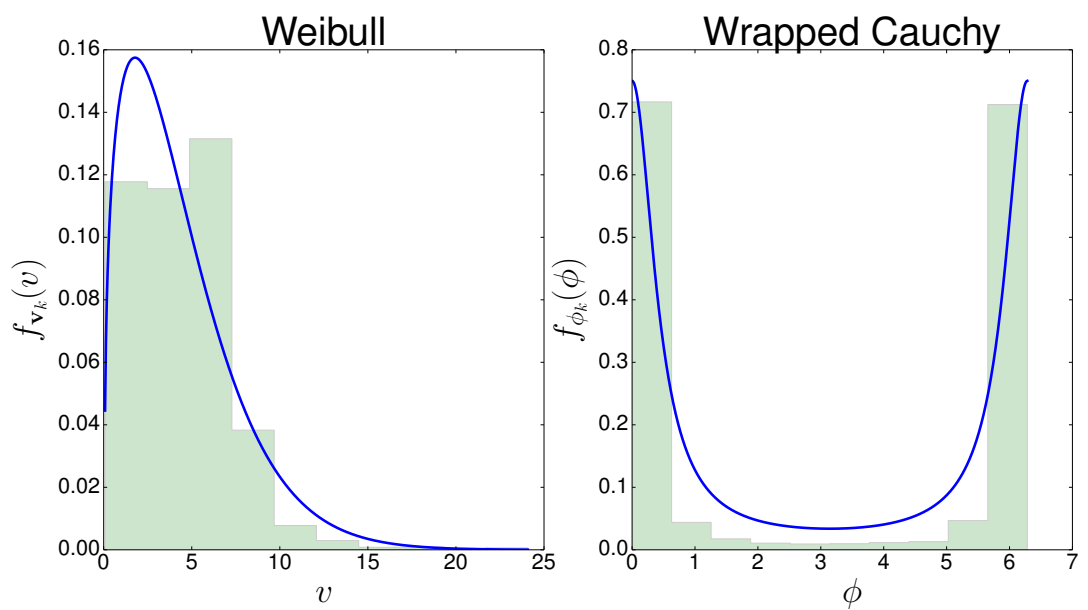

Fig. 3. Comparisons between the probability density functions of $\mathbf{v}_{k}$ and $\phi_{k}$ under 37) and the histograms obtained from the GPS data.

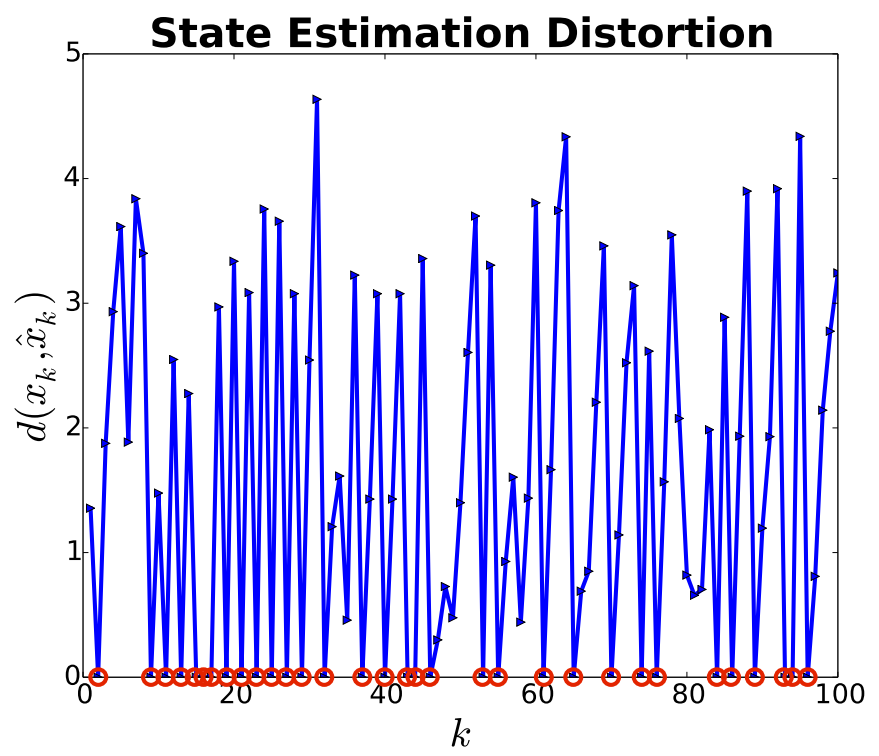

Fig. 4. State estimation distortion of the optimal remote estimation scheme

\section{APPENDIX}

\section{A. On Product Metric Space}

Given a metric space $(\mathbb{X}, d)$, we define a metric $\bar{d}$ on the product space $\mathbb{X}^{k}$ as follows: For $x_{1: k}=\left(x_{1}, \cdots, x_{k}\right)$ and $y_{1: k}=\left(y_{1}, \cdots, y_{k}\right)$ in $\mathbb{X}^{k}$

$$
\bar{d}\left(x_{1: k}, y_{1: k}\right)=\left[d^{2}\left(x_{1}, y_{1}\right)+\cdots+d^{2}\left(x_{k}, y_{k}\right)\right]^{1 / 2}
$$

Note that $\left(\mathbb{X}^{k}, \bar{d}\right)$ is a (product) metric space. For $(\mathbb{X}, d)$ and $\left(\mathbb{X}^{k}, \bar{d}\right)$, the following are true:

(F1) Let $\left\{\mathbb{K}_{j}\right\}_{j=1}^{k}$ be a collection of compact subsets of $\mathbb{X}$. The product set $\mathbb{K}_{1} \times \cdots \times \mathbb{K}_{k}$ is a compact subset of 
$\mathbb{X}^{k}$

(F2) Consider a sequence $\left\{x_{1: k}^{(i)}\right\}_{i \in \mathbb{N}}$ in $\mathbb{X}^{k}$. The sequence converges to $x_{1: k}$ in $\mathbb{X}^{k}$, i.e.,

$$
\lim _{i \rightarrow \infty} \bar{d}\left(x_{1: k}^{(i)}, x_{1: k}\right)=0
$$

if and only if $\left\{x_{j}^{(i)}\right\}_{i \in \mathbb{N}}$ converges to $x_{j}$ for all $j$ in $\{1, \cdots, k\}$, i.e.,

$$
\lim _{i \rightarrow \infty} d\left(x_{j}^{(i)}, x_{j}\right)=0
$$

holds for all $j$ in $\{1, \cdots, k\}$.

As a consequence of (F2) a function $\mathcal{G}: \mathbb{X}^{k} \rightarrow \mathbb{R}$ is continuous at $x_{1: k} \in \mathbb{X}^{k}$ if for any sequence $\left\{x_{1: k}^{(i)}\right\}_{i \in \mathbb{N}}$ for which $\lim _{i \rightarrow \infty} d\left(x_{j}^{(i)}, x_{j}\right)=0$ holds for all $j$ in $\{1, \cdots, k\}$, it holds that $\lim _{i \rightarrow \infty}\left|\mathcal{G}\left(x_{1: k}^{(i)}\right)-\mathcal{G}\left(x_{1: k}\right)\right|=0$.

\section{B. On Randomized Policies}

Let $\left(\boldsymbol{\tau}_{k}, \mathbf{x}_{\boldsymbol{\tau}_{k}}, \mathbf{x}_{k}\right) \mapsto \mathcal{T}_{k}\left(\boldsymbol{\tau}_{k}, \mathbf{x}_{\tau_{k}}, \mathbf{x}_{k}\right)$ be a randomized transmission policy defined in Section $\Pi$ that dictates the random variable $\mathbf{R}_{k}$ as in (4). Given a realization $\left(\tau_{k}, x_{\tau_{k}}, x_{k}\right)$ of $\left(\boldsymbol{\tau}_{k}, \mathbf{x}_{\tau_{k}}, \mathbf{x}_{k}\right)$, the variable $\mathbf{R}_{k}$ satisfies

$$
\mathbf{R}_{k}= \begin{cases}0 & \text { with probability } \mathbb{P}\left(\mathcal{T}_{k}\left(\boldsymbol{\tau}_{k}, \boldsymbol{x}_{\boldsymbol{\tau}_{k}}, \mathbf{x}_{k}\right)=0 \mid \boldsymbol{\tau}_{k}=\tau_{k}, \boldsymbol{x}_{\boldsymbol{\tau}_{k}}=x_{\tau_{k}}, \mathbf{x}_{k}=x_{k}\right) \\ 1 & \text { with probability } \mathbb{P}\left(\mathcal{T}_{k}\left(\boldsymbol{\tau}_{k}, \boldsymbol{x}_{\boldsymbol{\tau}_{k}}, \mathbf{x}_{k}\right)=1 \mid \boldsymbol{\tau}_{k}=\tau_{k}, \boldsymbol{x}_{\boldsymbol{\tau}_{k}}=x_{\tau_{k}}, \mathbf{x}_{k}=x_{k}\right)\end{cases}
$$

Let $\mathbf{x}_{j} \mapsto \mathcal{P}_{j}\left(\mathbf{x}_{j}\right)$ be a randomized policy defined in Section $\left[11\right.$ that dictates the random variable $\mathbf{R}_{j}$ as in 13 . Given a realization $x_{j}$ of $\mathbf{x}_{j}$, the variable $\mathbf{R}_{j}$ satisfies

$$
\mathbf{R}_{j}= \begin{cases}0 & \text { with probability } \mathbb{P}\left(\mathcal{P}_{j}\left(\mathbf{x}_{j}\right)=0 \mid \mathbf{x}_{j}=x_{j}\right) \\ 1 & \text { with probability } \mathbb{P}\left(\mathcal{P}_{j}\left(\mathbf{x}_{j}\right)=1 \mid \mathbf{x}_{j}=x_{j}\right)\end{cases}
$$

Throughout the work, we restrict our attention to the policies in which

$$
\mathbb{P}\left(\mathcal{P}_{j}\left(\mathbf{x}_{j}\right)=0 \mid \mathbf{x}_{j}=x_{j}\right)
$$

is a measurable function of $x_{j}$ on the measurable space $\left(\mathbb{R}^{2} \times[0,2 \pi), \mathfrak{B}\right)$ where $\mathfrak{B}$ is a Borel $\sigma$-algebra on $\mathbb{R}^{2} \times[0,2 \pi)$. As a case in point, consider a (deterministic) policy defined by

$$
\mathcal{P}_{j}\left(\mathbf{x}_{j}\right)= \begin{cases}0 & \text { if } \mathbf{x}_{j} \in \mathbb{D}_{j} \\ 1 & \text { otherwise }\end{cases}
$$

where $\mathbb{D}_{j} \in \mathfrak{B}$. It can be verified that

$$
\mathbb{P}\left(\mathcal{P}_{j}\left(\mathbf{x}_{j}\right)=0 \mid \mathbf{x}_{j}=x_{j}\right)= \begin{cases}0 & \text { if } x_{j} \in \mathbb{D}_{j} \\ 1 & \text { otherwise }\end{cases}
$$

is a measurable function of $x_{j}$. 


\section{Preliminary Concepts in Probability Theory}

We first review some of key definitions and results from probability theory [10], [12]. Let $(\mathbb{X}, d)$ be a complete separable metric space, and let $\mathcal{T}$ and $\mathfrak{B}$ be a topology and a Borel $\sigma$-algebra derived from the metric, respectively.

Definition C.1: Let $\mu$ be a probability measure on $(\mathbb{X}, \mathfrak{B})$. The probability measure is said to be tight if for every positive constant $\epsilon$, there exists a compact subset $\mathbb{K}$ of $(\mathbb{X}, \mathcal{T})$ for which $\mu(\mathbb{K})>1-\epsilon$ holds.

The following is adopted from Theorem 7.1.4 in [10].

Lemma C.2: Any probability measure $\mu$ on $(\mathbb{X}, \mathfrak{B})$ is tight.

Definition C.3: A probability measure $\mu$ defined on $(\mathbb{X}, \mathfrak{B})$ is said to be closed regular if for every $\mathbb{A}$ in $\mathfrak{B}$, it holds that

$$
\mu(\mathbb{A})=\sup \{\mu(\mathbb{F}) \mid \mathbb{F} \in \mathfrak{B} \text { closed, } \mathbb{F} \subset \mathbb{A}\}
$$

From Theorem 7.1.3 in [10], we can state the following Lemma.

Lemma C.4: Any probability measure $\mu$ on $(\mathbb{X}, \mathfrak{B})$ is closed regular.

Remark C.5: Let $\mu$ be a closed regular probability measure defined on $(\mathbb{X}, \mathfrak{B})$ and let $\mathbb{A}$ be a measurable subset in $\mathfrak{B}$. For every positive constant $\epsilon$, there exists a closed set $\mathbb{F}$ for which $\mathbb{F} \subset \mathbb{X} \backslash \mathbb{A}$ and $\mu(\mathbb{X} \backslash \mathbb{A})<\mu(\mathbb{F})+\epsilon$. Let us define an open set $\mathbb{O}=\mathbb{X} \backslash \mathbb{F}$. We can see that $\mathbb{O}$ satisfies $\mathbb{O} \supset \mathbb{A}$ and $\mu(\mathbb{O})<\mu(\mathbb{A})+\epsilon$. Hence we conclude that

$$
\mu(\mathbb{A})=\inf \{\mu(\mathbb{O}) \mid \mathbb{O} \in \mathfrak{B} \text { open, } \mathbb{O} \supset \mathbb{A}\}
$$

Definition C.6 (Convergence of Probability Measures): Let $\left\{\mu^{(i)}\right\}_{i \in \mathbb{N}}$ and $\mu$ be a sequence of probability measures and a probability measure defined on $(\mathbb{X}, \mathfrak{B})$, respectively, and let $\mathcal{C}_{b}(\mathbb{X})$ be the set of all bounded, continuous, real-valued functions on $\mathbb{X}$. The sequence is said to converge to $\mu$ if it holds that

$$
\lim _{i \rightarrow \infty} \int g \mathrm{~d} \mu^{(i)}=\int g \mathrm{~d} \mu
$$

for every $g$ in $\mathcal{C}_{b}(\mathbb{X})$. We denote the convergence by $\mu^{(i)} \rightarrow \mu$.

Definition C.7: Let $\left\{\mu^{(i)}\right\}_{i \in \mathbb{N}}$ be a sequence of probability measures defined on $(\mathbb{X}, \mathfrak{B})$. The probability measures are said to be uniformly tight if for every positive constant $\epsilon$, there exists a compact subset $\mathbb{K}$ of $(\mathbb{X}, \mathcal{T})$ for which $\mu^{(i)}(\mathbb{K})>1-\epsilon$ holds for all $i$ in $\mathbb{N}$.

A measurable subset $\mathbb{A}$ of $\mathbb{X}$ is said to be a $\mu$-continuity set if its boundary set has the zero measure with respect to $\mu$, i.e., $\mu(\operatorname{bd}(\mathbb{A}))=0$. The following is the portmanteau theorem (See Theorem 11.1.1 in [10]).

Theorem C.8: For a sequence $\left\{\mu^{(i)}\right\}_{i \in \mathbb{N}}$ of probability measures and a probability measure $\mu$ on $(\mathbb{X}, \mathfrak{B})$, the following are equivalent:

1) $\mu^{(i)} \rightarrow \mu$

2) $\lim \sup _{i \rightarrow \infty} \mu^{(i)}(\mathbb{F}) \leq \mu(\mathbb{F})$ for any closed subset $\mathbb{F}$ of $\mathbb{X}$

3) $\liminf _{i \rightarrow \infty} \mu^{(i)}(\mathbb{O}) \geq \mu(\mathbb{O})$ for any open subset $\mathbb{O}$ of $\mathbb{X}$

4) $\lim _{i \rightarrow \infty} \mu^{(i)}(\mathbb{A})=\mu(\mathbb{A})$ for any $\mu$-continuity subset $\mathbb{A}$ of $\mathbb{X}$. 


\section{Preliminary Results}

Lemma D.9: The metric space $\left(\mathbb{R}^{2} \times[0,2 \pi), d\right)$ defined in Section $\mathrm{II}$ is complete, separable, and proper.

Lemma D.10: Given estimates $\hat{x}_{k: N}$, let $\mathcal{P}_{k: N}$ be non-degenerate policies that belong to $\mathfrak{P}\left(\hat{x}_{k: N}\right)$. The following are true for all $j$ in $\{k, \cdots, N\}$ :

1) $\mathbb{P}\left(\mathbf{x}_{j} \in \overline{\mathbb{D}}_{j} \mid \mathbf{R}_{k}=0, \cdots, \mathbf{R}_{j}=0\right)=1$

2) $\mathbb{P}\left(\mathbf{x}_{j} \in \underline{\mathbb{D}}_{j} \mid \mathbf{R}_{k}=0, \cdots, \mathbf{R}_{j}=1\right)=0$ subject to $\mathbf{R}_{j}=\mathcal{P}_{j}\left(\mathbf{x}_{j}\right)$ for each $j$ in $\{k, \cdots, N\}$, where $\overline{\mathbb{D}}_{j}$ and $\underline{\mathbb{D}}_{j}$ are defined in 19$\}$.

The proof directly follows from Proposition III.9.

Based on Lemma D.10, we can state the following proposition.

Proposition D.11: Given estimates $\hat{x}_{k: N}$, let $\mathcal{P}_{k: N}$ be non-degenerate policies that belong to $\mathfrak{P}\left(\hat{x}_{k: N}\right)$. Consider compact sets $\left\{\mathbb{K}_{j}\right\}_{j=k}^{N}$ given by ${ }^{14}$

$$
\mathbb{K}_{j}=\left\{x \in \mathbb{R}^{2} \times[0,2 \pi) \mid d^{2}\left(x, \hat{x}_{j}\right) \leq c_{j}^{\prime}\right\}
$$

The following holds for all $j$ in $\{k, \cdots, N\}$ :

$$
\mathbb{P}\left(\mathbf{x}_{j} \in \mathbb{K}_{j} \mid \mathbf{R}_{k}=0, \cdots, \mathbf{R}_{j}=0\right)=1
$$

subject to $\mathbf{R}_{j}=\mathcal{P}_{j}\left(\mathbf{x}_{j}\right)$ for each $j$ in $\{k, \cdots, N\}$.

The proof follows from the fact that $\mathbb{K}_{j}$ contains the set $\overline{\mathbb{D}}_{j}$ defined in $19 \mathrm{a}$ and Lemma D.10.

Proposition D.12: Given policies $\mathcal{P}_{k: N}$, for each $j$ in $\{k, \cdots, N\}$, let us define

$$
\begin{aligned}
\mu_{j \mid j}(\mathbb{A}) & =\mathbb{P}\left(\mathbf{x}_{j} \in \mathbb{A} \mid \mathbf{R}_{k}=0, \cdots, \mathbf{R}_{j}=0\right) \\
\mu_{j \mid j-1}(\mathbb{A}) & =\mathbb{P}\left(\mathbf{x}_{j} \in \mathbb{A} \mid \mathbf{R}_{k}=0, \cdots, \mathbf{R}_{j-1}=0\right)
\end{aligned}
$$

subject to $\mathbf{R}_{j}=\mathcal{P}_{j}\left(\mathbf{x}_{j}\right)$ for each $j$ in $\{k, \cdots, N\}$, where $\mathbb{A}$ is a Borel-measurable subset. The probability measures (43) evolve according to the following update rules:

1) Policy update rule:

$$
\mu_{j \mid j}(\mathbb{A})=\frac{\int_{\mathbb{A}} \mathbb{P}\left(\mathcal{P}_{j}\left(\mathbf{x}_{j}\right)=0 \mid \mathbf{x}_{j}=x\right) \mathrm{d} \mu_{j \mid j-1}}{\mathbb{P}\left(\mathbf{R}_{j}=0 \mid \mathbf{R}_{k}=0, \cdots, \mathbf{R}_{j-1}=0\right)}
$$

provided that $\mathbb{P}\left(\mathbf{R}_{j}=0 \mid \mathbf{R}_{k}=0, \cdots, \mathbf{R}_{j-1}=0\right)$ is positive.

2) Process update rule:

$$
\mu_{j \mid j-1}(\mathbb{A})=\int_{\mathbb{R}^{2} \times[0,2 \pi)} \mathbb{P}\left(\mathbf{x}_{j} \in \mathbb{A} \mid \mathbf{x}_{j-1}=x\right) \mathrm{d} \mu_{j-1 \mid j-1}
$$

\footnotetext{
${ }^{14}$ Due to the properness of the metric space $\left(\mathbb{R}^{2} \times[0,2 \pi), d\right)$ (see Lemma D.9, every closed ball is a compact set.
} 


\section{E. Proof of Proposition III.13}

To start with, we note that for each $j$ in $\{k, \cdots, N\}$, the function $\mathcal{G}_{j}$ can be written as follows:

$$
\mathcal{G}_{j}\left(x_{j-1}, \hat{x}_{j: N}\right)=\mathbb{E}_{\mathbf{x}_{j}}\left[g_{j}\left(\mathbf{x}_{j}, \hat{x}_{j: N}\right) \mid \mathbf{x}_{j-1}=x_{j-1}\right]
$$

with $\mathcal{G}_{N+1}=0$, where $g_{j}\left(x_{j}, \hat{x}_{j: N}\right)=\min \left\{d^{2}\left(x_{j}, \hat{x}_{j}\right)+\mathcal{G}_{j+1}\left(x_{j}, \hat{x}_{j+1: N}\right), c_{j}^{\prime}\right\}$.

We prove the statement using mathematical induction starting from $j=N+1$. Since $\mathcal{G}_{N+1}$ is constant, e.g., $\mathcal{G}_{N+1}=0$, it is a continuous function. Now suppose that $\mathcal{G}_{j+1}$ is a continuous function. Note that $g_{j}$ in (44) is a continuous function. To verify the continuity of $\mathcal{G}_{j}$, let $\left\{x_{j-1}^{(i)}\right\}_{i \in \mathbb{N}}$ and $\left\{\hat{x}_{j: N}^{(i)}\right\}_{i \in \mathbb{N}}$ be sequences that converge to $x_{j-1}$ and $\hat{x}_{j: N}$, respectively. For each set $\mathbb{A}$ in $\mathfrak{B}$, let us define

$$
\begin{aligned}
\mu_{j}^{(i)}(\mathbb{A}) & =\mathbb{P}\left(\mathbf{x}_{j} \in \mathbb{A} \mid \mathbf{x}_{j-1}=x_{j-1}^{(i)}\right) \\
\mu_{j}(\mathbb{A}) & =\mathbb{P}\left(\mathbf{x}_{j} \in \mathbb{A} \mid \mathbf{x}_{j-1}=x_{j-1}\right)
\end{aligned}
$$

By Assumption II.4 and Theorem C.8. we can see that $\left\{\mu_{j}^{(i)}\right\}_{i \in \mathbb{N}}$ converges to $\mu_{j}$.

Since $\left(\mathbb{R}^{2} \times[0,2 \pi), d\right)$ is a complete, separable metric space by Lemma D.9 using the Skorokhod representation theorem [13], we can see that there is a sequence of random variables $\left\{\mathbf{y}_{j}^{(i)}\right\}_{i \in \mathbb{N}}$ and a random variable $\mathbf{y}_{j}$ all defined on a common probability space $(\Omega, \mathfrak{F}, \nu)$ in which the following three facts are true:

(F1) $\mu_{j}^{(i)}$ is the probability measure of $\mathbf{y}_{j}^{(i)}$, i.e., $\nu\left(\left\{\omega \in \Omega \mid \mathbf{y}_{j}^{(i)}(\omega) \in \mathbb{A}\right\}\right)=\mu_{j}^{(i)}(\mathbb{A})$ for each $\mathbb{A}$ in $\mathfrak{B}$.

(F2) $\mu_{j}$ is the probability measure of $\mathbf{y}_{j}$, i.e., $\nu\left(\left\{\omega \in \Omega \mid \mathbf{y}_{j}(\omega) \in \mathbb{A}\right\}\right)=\mu_{j}(\mathbb{A})$ for each $\mathbb{A}$ in $\mathfrak{B}$.

(F3) $\left\{\mathbf{y}_{j}^{(i)}\right\}_{i \in \mathbb{N}}$ converges to $\mathbf{y}_{j}$ almost surely.

From (F1) and (F2), we can derive

$$
\begin{aligned}
& \mathcal{G}_{j}\left(x_{j-1}^{(i)}, \hat{x}_{j: N}^{(i)}\right)-\mathcal{G}_{j}\left(x_{j-1}, \hat{x}_{j: N}\right) \\
& =\mathbb{E}_{\mathbf{x}_{j}}\left[g_{j}\left(\mathbf{x}_{j}, \hat{x}_{j: N}^{(i)}\right) \mid \mathbf{x}_{j-1}=x_{j-1}^{(i)}\right]-\mathbb{E}_{\mathbf{x}_{j}}\left[g_{j}\left(\mathbf{x}_{j}, \hat{x}_{j: N}\right) \mid \mathbf{x}_{j-1}=x_{j-1}\right] \\
& =\int_{\Omega} g_{j}\left(\mathbf{y}_{j}^{(i)}(\omega), \hat{x}_{j: N}^{(i)}\right) \mathrm{d} \nu-\int_{\Omega} g_{j}\left(\mathbf{y}_{j}(\omega), \hat{x}_{j: N}\right) \mathrm{d} \nu
\end{aligned}
$$

Notice that by the fact that $c_{j}^{\prime}$ is a fixed constant and $g$ is a non-negative function, it holds that

$$
0 \leq g_{j}\left(\mathbf{y}_{j}^{(i)}(\omega), \hat{x}_{j: N}^{(i)}\right) \leq c_{j}^{\prime}
$$

for every $i$ in $\mathbb{N}$ and every $\omega \in \Omega$. Hence, the sequence of functions $\left\{g_{j}\left(\mathbf{y}_{j}^{(i)}(\cdot), \hat{x}_{j: N}^{(i)}\right)\right\}_{i \in \mathbb{N}}$ is uniformly bounded. Also, by the continuity of $g_{j}$ and (F3), it holds that

$$
\lim _{i \rightarrow \infty} g_{j}\left(\mathbf{y}_{j}^{(i)}(\omega), \hat{x}_{j: N}^{(i)}\right)=g_{j}\left(\mathbf{y}_{j}(\omega), \hat{x}_{j: N}\right)
$$

for almost every $\omega$ in $\Omega$. Using the bounded convergence theorem (see Theorem 16.5 in [12]), we have that

$$
\begin{aligned}
& \lim _{i \rightarrow \infty}\left|\mathcal{G}_{j}\left(x_{j-1}^{(i)}, \hat{x}_{j: N}^{(i)}\right)-\mathcal{G}_{j}\left(x_{j-1}, \hat{x}_{j: N}\right)\right| \\
& =\lim _{i \rightarrow \infty}\left|\int_{\Omega} g_{j}\left(\mathbf{y}_{j}^{(i)}(\omega), \hat{x}_{j: N}^{(i)}\right) \mathrm{d} \nu-\int_{\Omega} g_{j}\left(\mathbf{y}_{j}(\omega), \hat{x}_{j: N}\right) \mathrm{d} \nu\right|=0
\end{aligned}
$$

which proves that the function $\mathcal{G}_{j}$ is continuous.

Finally, by induction, we conclude that the functions $\left\{\mathcal{G}_{j}\right\}_{j=k}^{N}$ are all continuous. 
F. Proofs of Proposition III.14 and Lemma III.16

Lemma F.13: For each $j$ in $\{k, \cdots, N\}$, there exist estimates $\hat{x}_{j: N}$ for which the set given by

$$
\underline{\mathbb{D}}_{j}=\left\{x_{j} \in \mathbb{R}^{2} \times[0,2 \pi) \mid d^{2}\left(x_{j}, \hat{x}_{j}\right)+\mathbb{E}_{\mathbf{x}_{j+1}}\left[J_{j+1}^{*}\left(\mathbf{x}_{j+1}, \hat{x}_{j+1: N}\right) \mid \mathbf{x}_{j}=x_{j}\right]<c_{j}^{\prime}\right\}
$$

is non-empty, where $J_{j+1}^{*}$ is defined in (18).

Proof: Recall how $c_{j}^{\prime}$ is determined by (9) with the solutions $\mathcal{T}_{j+1: N}^{<j>}$ and $\mathcal{E}_{j+1: N}^{<j>}$ to Sub-problem $j+1$. Let us select a fixed point $x_{j}^{o}$ in $\mathbb{R}^{2} \times[0,2 \pi)$. Under the choice of $\hat{x}_{j}=x_{j}^{o}$ and $\hat{x}_{l}=\mathcal{E}_{l}^{<j>}\left(x_{j}\right)$ for each $l$ in $\{j+1, \cdots, N\}$, by a similar argument as in Remark III.2, we can see that

$$
\mathbb{E}_{\mathbf{x}_{j+1}}\left[J_{j+1}^{*}\left(\mathbf{x}_{j+1}, \hat{x}_{j+1: N}\right) \mid \mathbf{x}_{j}=x_{j}\right]=\mathbb{E}_{\mathbf{x}_{j+1}}\left[J_{j+1}^{*}\left(\mathbf{x}_{j+1}, \hat{x}_{j+1: N}^{\prime}\right) \mid \mathbf{x}_{j}=0\right]
$$

where $\hat{x}_{l}^{\prime}=\mathcal{E}_{l}^{<j>}(0)$ for each $l$ in $\{j+1, \cdots, N\}$. Hence, at $x_{j}=x_{j}^{o}$, it holds that

$$
\begin{aligned}
& d^{2}\left(x_{j}, \hat{x}_{j}\right)+\mathbb{E}_{\mathbf{x}_{j+1}}\left[J_{j+1}^{*}\left(\mathbf{x}_{j+1}, \hat{x}_{j+1: N}\right) \mid \mathbf{x}_{j}=x_{j}\right] \\
& =\mathbb{E}_{\mathbf{x}_{j+1}}\left[J_{j+1}^{*}\left(\mathbf{x}_{j+1}, \hat{x}_{j+1: N}\right) \mid \mathbf{x}_{j}=x_{j}\right] \\
& <c_{j}+\mathbb{E}_{\mathbf{x}_{j+1}}\left[J_{j+1}^{*}\left(\mathbf{x}_{j+1}, \hat{x}_{j+1: N}\right) \mid \mathbf{x}_{j}=x_{j}\right] \\
& =c_{j}+\mathbb{E}_{\mathbf{x}_{j+1}}\left[J_{j+1}^{*}\left(\mathbf{x}_{j+1}, \hat{x}_{j+1: N}^{\prime}\right) \mid \mathbf{x}_{j}=0\right]=c_{j}^{\prime}
\end{aligned}
$$

This proves the Lemma.

Lemma F.14: Given estimates $\hat{x}_{k: N}$, the sets $\overline{\mathbb{D}}_{j}$ and $\underline{\mathbb{D}}_{j}$ defined in (19) are closed and open, respectively, for all $j$ in $\{k, \cdots, N\}$.

The proof directly follows from the continuity of the functions $\left\{\mathcal{G}_{j}\right\}_{j=k}^{N}$, each defined in 23] (See Proposition III.13).

Proof of Proposition III.14. By contradiction, suppose that the degenerate policies $\mathcal{P}_{k: N}^{*}$ and estimates $\hat{x}_{k: N}^{*}$ are jointly optimal for (12). Let $j_{0} \in\{k, \cdots, N\}$ be the smallest integer for which

$$
\mathbb{P}\left(\mathbf{R}_{j_{0}}^{*}=0 \mid \mathbf{R}_{k}^{*}=0, \cdots, \mathbf{R}_{j_{0}-1}^{*}=0\right)=0
$$

holds subject to $\mathbf{R}_{j}^{*}=\mathcal{P}_{j}^{*}\left(\mathbf{x}_{j}\right)$ for each $j$ in $\{k, \cdots, N\}$. Since $j_{0}$ is the smallest such integer, by Proposition D.12. the probability measure $\mu_{j_{0} \mid j_{0}-1}$ of $\mathbf{x}_{j_{0}}$ is well-defined.

Using Lemma F.13, let us choose $\hat{x}_{j_{0}: N}^{\circ} \in\left(\mathbb{R}^{2} \times[0,2 \pi)\right)^{N-j_{0}+1}$ for which the set given by

$$
\underline{\mathbb{D}}_{j_{0}}^{\circ}=\left\{x_{j_{0}} \in \mathbb{R}^{2} \times[0,2 \pi) \mid d^{2}\left(x_{j_{0}}, \hat{x}_{j_{0}}^{\circ}\right)+\mathbb{E}_{\mathbf{x}_{j_{0}+1}}\left[J_{j_{0}+1}^{*}\left(\mathbf{x}_{j_{0}+1}, \hat{x}_{j_{0}+1: N}^{\circ}\right) \mid \mathbf{x}_{j_{0}}=x_{j_{0}}\right]<c_{j_{0}}^{\prime}\right\}
$$

is non-empty. Note that according to Lemma F.14 the set $\mathbb{D}_{j}^{\circ}$ is open; hence, from Assumption II.4 and Proposition D.12, we have that

$$
\mathbb{P}\left(\mathbf{x}_{j_{0}} \in \underline{\mathbb{D}}_{j_{0}}^{\circ} \mid \mathbf{R}_{k}^{*}=0, \cdots, \mathbf{R}_{j_{0}-1}^{*}=0\right)>0
$$

For each $j$ in $\left\{j_{0}, \cdots, N\right\}$, let us define a function $\mathcal{P}_{j}^{\circ}: \mathbb{R}^{2} \times[0,2 \pi) \rightarrow\{0,1\}$ as follows:

$$
\mathcal{P}_{j}^{\circ}\left(x_{j}\right)= \begin{cases}0 & \text { if } x_{j} \in \underline{\mathbb{D}}_{j}^{\circ} \\ 1 & \text { otherwise }\end{cases}
$$


where

$$
\underline{\mathbb{D}}_{j}^{\circ}=\left\{x_{j} \in \mathbb{R}^{2} \times[0,2 \pi) \mid d^{2}\left(x_{j}, \hat{x}_{j}^{\circ}\right)+\mathbb{E}_{\mathbf{x}_{j+1}}\left[J_{j+1}^{*}\left(\mathbf{x}_{j+1}, \hat{x}_{j+1: N}^{\circ}\right) \mid \mathbf{x}_{j}=x_{j}\right]<c_{j}^{\prime}\right\}
$$

Let us select new policies $\mathcal{P}_{k: N}^{\prime}$ and estimates $\hat{x}_{k: N}^{\prime}$ as follows: for each $j$ in $\{k, \cdots, N\}$,

$$
\begin{aligned}
& \mathcal{P}_{j}^{\prime}= \begin{cases}\mathcal{P}_{j}^{*} & \text { if } j \in\left\{k, \cdots, j_{0}-1\right\} \\
\mathcal{P}_{j}^{\circ} & \text { if } j \in\left\{j_{0}, \cdots, N\right\}\end{cases} \\
& \hat{x}_{j}^{\prime}= \begin{cases}\hat{x}_{j}^{*} & \text { if } j \in\left\{k, \cdots, j_{0}-1\right\} \\
\hat{x}_{j}^{\circ} & \text { if } j \in\left\{j_{0}, \cdots, N\right\}\end{cases}
\end{aligned}
$$

By definition, under the new policies $\mathcal{P}_{k: N}^{\prime}$, it holds that

$$
\mathbb{P}\left(\mathbf{x}_{j_{0}} \in \underline{\mathbb{D}}_{j_{0}}^{\circ} \mid \mathbf{R}_{k}^{\prime}=0, \cdots, \mathbf{R}_{j_{0}}^{\prime}=1\right)=0
$$

subject to $\mathbf{R}_{j}^{\prime}=\mathcal{P}_{j}^{\prime}\left(\mathbf{x}_{j}\right)$ for each $j$ in $\{k, \cdots, N\}$. This implies that

$$
\begin{aligned}
& \mathbb{P}\left(\mathbf{x}_{j_{0}} \in \underline{\mathbb{D}}_{j_{0}}^{\circ} \mid \mathbf{R}_{k}^{\prime}=0, \cdots, \mathbf{R}_{j_{0}-1}^{\prime}=0\right) \\
& =\mathbb{P}\left(\mathbf{x}_{j_{0}} \in \underline{\mathbb{D}}_{j_{0}}^{\circ} \mid \mathbf{R}_{k}^{\prime}=0, \cdots, \mathbf{R}_{j_{0}}^{\prime}=0\right) \cdot \mathbb{P}\left(\mathbf{R}_{j_{0}}^{\prime}=0 \mid \mathbf{R}_{k}^{\prime}=0, \cdots, \mathbf{R}_{j_{0}-1}^{\prime}=0\right)
\end{aligned}
$$

By (51), 52a), and (54), we can see that

$$
\mathbb{P}\left(\mathbf{R}_{j_{0}}^{\prime}=0 \mid \mathbf{R}_{k}^{\prime}=0, \cdots, \mathbf{R}_{j_{0}-1}^{\prime}=0\right)>0
$$

Due to 49, by Remark III.8, we can see that

$$
\mathbb{E}_{\mathbf{x}_{j_{0}}}\left[J_{j_{0}}\left(\mathbf{x}_{j_{0}}, \mathcal{P}_{j_{0}: N}^{*}, \hat{x}_{j_{0}: N}^{*}\right) \mid \mathbf{R}_{k}^{*}=0, \cdots, \mathbf{R}_{j_{0}-1}^{*}=0\right]=c_{j_{0}}^{\prime}
$$

While, by (14), 52], and 55], we can see that

$$
\begin{aligned}
& \mathbb{E}_{\mathbf{x}_{j_{0}}}\left[J_{j_{0}}\left(\mathbf{x}_{j_{0}}, \mathcal{P}_{j_{0}: N}^{\prime}, \hat{x}_{j_{0}: N}^{\prime}\right) \mid \mathbf{R}_{k}^{\prime}=0, \cdots, \mathbf{R}_{j_{0}-1}^{\prime}=0\right] \\
& =\mathbb{E}_{\mathbf{x}_{j_{0}}}\left[J_{j_{0}}^{*}\left(\mathbf{x}_{j_{0}}, \hat{x}_{j_{0}: N}^{\prime}\right) \mid \mathbf{R}_{k}^{\prime}=0, \cdots, \mathbf{R}_{j_{0}-1}^{\prime}=0\right]<c_{j_{0}}^{\prime}
\end{aligned}
$$

These relations imply that

$$
\begin{aligned}
& \mathbb{E}_{\mathbf{x}_{j_{0}}}\left[J_{j_{0}}\left(\mathbf{x}_{j_{0}}, \mathcal{P}_{j_{0}: N}^{\prime}, \hat{x}_{j_{0}: N}^{\prime}\right) \mid \mathbf{R}_{k}^{\prime}=0, \cdots, \mathbf{R}_{j_{0}-1}^{\prime}=0\right] \\
& <\mathbb{E}_{\mathbf{x}_{j_{0}}}\left[J_{j_{0}}\left(\mathbf{x}_{j_{0}}, \mathcal{P}_{j_{0}: N}^{*}, \hat{x}_{j_{0}: N}^{*}\right) \mid \mathbf{R}_{k}^{*}=0, \cdots, \mathbf{R}_{j_{0}-1}^{*}=0\right]
\end{aligned}
$$

Using the facts that $\mathcal{P}_{j}^{\prime}=\mathcal{P}_{j}^{*}$ and $\hat{x}_{j}^{\prime}=\hat{x}_{j}^{*}$ for each $j$ in $\left\{k, \cdots, j_{0}-1\right\}$, and $j_{0}$ is the smallest integer for which (49) holds, from (14), we can infer that

$$
\mathbb{E}_{\mathbf{x}_{k}}\left[J_{k}\left(\mathbf{x}_{k}, \mathcal{P}_{k: N}^{\prime}, \hat{x}_{k: N}^{\prime}\right)\right]<\mathbb{E}_{\mathbf{x}_{k}}\left[J_{k}\left(\mathbf{x}_{k}, \mathcal{P}_{k: N}^{*}, \hat{x}_{k: N}^{*}\right)\right]
$$

which violates $\hat{x}_{k: N}^{*}$ is a global minimizer. Therefore, every global minimizer has to be non-degenerate. 
Lemma F.15: Consider policies $\left\{\mathcal{P}_{k: N}^{(i)}\right\}_{i \in \mathbb{N}}$ and estimates $\left\{\hat{x}_{k: N}^{(i)}\right\}_{i \in \mathbb{N}}$ satisfying $\mathcal{P}_{k: N}^{(i)} \in \mathfrak{P}\left(\hat{x}_{k: N}^{(i-1)}\right)$ for all $i$ in $\{k, \cdots, N\}$. Suppose that there exists a positive constant $\epsilon$ for which the following holds for all $i$ in $\mathbb{N}$ and $j$ in $\left\{k, \cdots, j_{0}\right\}$ :

$$
\mathbb{P}\left(\mathbf{R}_{j}^{(i)}=0 \mid \mathbf{R}_{k}^{(i)}=0, \cdots, \mathbf{R}_{j-1}^{(i)}=0\right) \geq \epsilon
$$

subject to $\mathbf{R}_{j}^{(i)}=\mathcal{P}_{j}^{(i)}\left(\mathbf{x}_{j}\right)$ for each $i$ in $\mathbb{N}$ and $j$ in $\left\{k, \cdots, j_{0}\right\}$. Then the sequence $\left\{\hat{x}_{j}^{(i)}\right\}_{i \in \mathbb{N}}$ is bounded for all $j$ in $\left\{k, \cdots, j_{0}\right\}$.

Proof: By contradiction, suppose that there exists $j^{\prime}$ in $\left\{k, \cdots, j_{0}\right\}$ such that for a subsequence $\left\{\hat{x}_{j^{\prime}}^{\left(i_{l}-1\right)}\right\}_{l \in \mathbb{N}}$ of $\left\{\hat{x}_{j^{\prime}}^{(i)}\right\}_{i \in \mathbb{N}}$, it holds that

$$
d\left(0, \hat{x}_{j^{\prime}}^{\left(i_{l}-1\right)}\right) \stackrel{l \rightarrow \infty}{\longrightarrow} \infty
$$

For each $l$ in $\mathbb{N}$, let us choose a compact set $\mathbb{K}_{j^{\prime}}^{\left(i_{l}\right)}=\left\{x \in \mathbb{R}^{2} \times[0,2 \pi) \mid d^{2}\left(x, \hat{x}_{j^{\prime}}^{\left(i_{l}-1\right)}\right) \leq c_{j}^{\prime}\right\}$. Then, according to Proposition D.11, the following holds for all $l$ in $\mathbb{N}$ :

$$
\mathbb{P}\left(\mathbf{x}_{j^{\prime}} \in \mathbb{K}_{j^{\prime}}^{\left(i_{l}\right)} \mid \mathbf{R}_{k}^{\left(i_{l}\right)}=0, \cdots, \mathbf{R}_{j^{\prime}}^{\left(i_{l}\right)}=0\right)=1
$$

subject to $\mathbf{R}_{j}^{\left(i_{l}\right)}=\mathcal{P}_{j}^{\left(i_{l}\right)}\left(\mathbf{x}_{j}\right)$ for each $l$ in $\mathbb{N}$ and $j$ in $\left\{k, \cdots, j^{\prime}\right\}$. Using [56, we can derive the following:

$$
\begin{aligned}
& \mathbb{P}\left(\mathbf{R}_{j^{\prime}}^{\left(i_{l}\right)}=0 \mid \mathbf{R}_{k}^{\left(i_{l}\right)}=0, \cdots, \mathbf{R}_{j^{\prime}-1}^{\left(i_{l}\right)}=0\right) \\
& =\frac{\mathbb{P}\left(\mathbf{R}_{j^{\prime}}^{\left(i_{l}\right)}=0 \mid \mathbf{x}_{j^{\prime}} \in \mathbb{K}_{j^{\prime}}^{\left(i_{l}\right)}, \mathbf{R}_{k}^{\left(i_{l}\right)}=0, \cdots, \mathbf{R}_{j^{\prime}-1}^{\left(i_{l}\right)}=0\right)}{\mathbb{P}\left(\mathbf{x}_{j^{\prime}} \in \mathbb{K}_{j^{\prime}}^{\left(i_{l}\right)} \mid \mathbf{R}_{k}^{\left(i_{l}\right)}=0, \cdots, \mathbf{R}_{j^{\prime}}^{\left(i_{l}\right)}=0\right)} \cdot \mathbb{P}\left(\mathbf{x}_{j^{\prime}} \in \mathbb{K}_{j^{\prime}}^{\left(i_{l}\right)} \mid \mathbf{R}_{k}^{\left(i_{l}\right)}=0, \cdots, \mathbf{R}_{j^{\prime}-1}^{\left(i_{l}\right)}=0\right) \\
& \leq \mathbb{P}\left(\mathbf{x}_{j^{\prime}} \in \mathbb{K}_{j^{\prime}}^{\left(i_{l}\right)} \mid \mathbf{R}_{k}^{\left(i_{l}\right)}=0, \cdots, \mathbf{R}_{j^{\prime}-1}^{\left(i_{l}\right)}=0\right) \\
& =\frac{\mathbb{P}\left(\mathbf{x}_{j^{\prime}} \in \mathbb{K}_{j^{\prime}}^{\left(i_{l}\right)}, \mathbf{R}_{k}^{\left(i_{l}\right)}=0, \cdots, \mathbf{R}_{j^{\prime}-1}^{\left(i_{l}\right)}=0\right)}{\prod_{j=k}^{j^{\prime}-1} \mathbb{P}\left(\mathbf{R}_{j}^{\left(i_{l}\right)}=0 \mid \mathbf{R}_{k}^{\left(i_{l}\right)}=0, \cdots, \mathbf{R}_{j-1}^{\left(i_{l}\right)}=0\right)} \\
& \leq \epsilon^{k-j^{\prime}} \cdot \mathbb{P}\left(\mathbf{x}_{j^{\prime}} \in \mathbb{K}_{j^{\prime}}^{\left(i_{l}\right)}\right)
\end{aligned}
$$

holds for all $l$ in $\mathbb{N}$. Hence, by Lemma C.2 and [57), we can see that

$$
\lim _{l \rightarrow \infty} \mathbb{P}\left(\mathbf{x}_{j^{\prime}} \in \mathbb{K}_{j^{\prime}}^{\left(i_{l}\right)}\right)=0
$$

In conjunction with [59), we conclude that

$$
\lim _{l \rightarrow \infty} \mathbb{P}\left(\mathbf{R}_{j^{\prime}}^{\left(i_{l}\right)}=0 \mid \mathbf{R}_{k}^{\left(i_{l}\right)}=0, \cdots, \mathbf{R}_{j^{\prime}-1}^{\left(i_{l}\right)}=0\right)=0
$$

This contradicts the fact that 56 holds for all $i$ in $\mathbb{N}$ and $j$ in $\left\{k, \cdots, j_{0}\right\}$.

Proof of Lemma III.16. For a positive real $r$, let us define

$$
\mathbb{K}_{r} \stackrel{\text { def }}{=}\left\{\hat{x}_{k: N} \in\left(\mathbb{R}^{2} \times[0,2 \pi)\right)^{N-k+1} \mid d\left(0, \hat{x}_{j}\right) \leq r \text { for all } j \text { in }\{k, \cdots, N\}\right\}
$$

To prove the lemma, it is sufficient to show that there exists $r>0$ for which with $\mathbb{K}=\mathbb{K}_{r}$, the statement of the lemma is true. By contradiction, suppose that there exists a sequence $\left\{\hat{x}_{k: N}^{(i)}\right\}_{i \in \mathbb{N}} \subset\left(\mathbb{R}^{2} \times[0,2 \pi)\right)^{N-k+1}$ that satisfies the following hypotheses: 
(H1) For each element $\hat{x}_{k: N}^{(i)}$ of the sequence, it holds that $\hat{x}_{k: N}^{(i)} \notin \mathbb{K}_{i}$.

(H2) For every $\hat{x}_{k: N}$ in $\mathbb{K}_{i}$, it holds that $\mathcal{G}\left(\hat{x}_{k: N}\right)>\mathcal{G}\left(\hat{x}_{k: N}^{(i)}\right)$.

We constructively prove that the hypothesis $(\mathbf{H 2})$ is violated for sufficiently large $i$ in $\mathbb{N}$. To proceed, let us select policies $\left\{\mathcal{P}_{k: N}^{(i)}\right\}_{i \in \mathbb{N}}$ that satisfy $\mathcal{P}_{k: N}^{(i)} \in \mathfrak{P}\left(\hat{x}_{k: N}^{(i-1)}\right)$. Let $j_{0} \in\{k, \cdots, N\}$ be the smallest integer for which there is a subsequence $\left\{\mathcal{P}_{k: j_{0}}^{\left(i_{l}\right)}\right\}_{l \in \mathbb{N}}$ of $\left\{\mathcal{P}_{k: j_{0}}^{(i)}\right\}_{i \in \mathbb{N}}$ satisfying 15

$$
\lim _{l \rightarrow \infty} \mathbb{P}\left(\mathbf{R}_{j_{0}}^{\left(i_{l}\right)}=0 \mid \mathbf{R}_{k}^{\left(i_{l}\right)}=0, \cdots, \mathbf{R}_{j_{0}-1}^{\left(i_{l}\right)}=0\right)=0
$$

subject to $\mathbf{R}_{j}^{\left(i_{l}\right)}=\mathcal{P}_{j}^{\left(i_{l}\right)}\left(\mathbf{x}_{j}\right)$ for each $l$ in $\mathbb{N}$ and $j$ in $\left\{k, \cdots, j_{0}\right\}$. Note from [14] and 63, we have that

$$
\lim _{l \rightarrow \infty} \mathbb{E}_{\mathbf{x}_{j_{0}}}\left[J_{j_{0}}\left(\mathbf{x}_{j_{0}}, \mathcal{P}_{j_{0}: N}^{\left(i_{l}\right)}, \hat{x}_{j_{0}: N}^{\left(i_{l}-1\right)}\right) \mid \mathbf{R}_{k}^{\left(i_{l}\right)}=0, \cdots, \mathbf{R}_{j_{0}-1}^{\left(i_{l}\right)}=0\right]=c_{j_{0}}^{\prime}
$$

Also, according to Lemma F.15 the sequence $\left\{\hat{x}_{j}^{(i)}\right\}_{i \in \mathbb{N}}$ is bounded for all $j$ in $\left\{k, \cdots, j_{0}-1\right\}$.

Using Lemma F.13, let us choose $\hat{x}_{j_{0}: N}^{\circ} \in\left(\mathbb{R}^{2} \times[0,2 \pi)\right)^{N-j_{0}+1}$ for which the set given by

$$
\underline{\mathbb{D}}_{j_{0}}^{\circ}=\left\{x_{j_{0}} \in \mathbb{R}^{2} \times[0,2 \pi) \mid d^{2}\left(x_{j_{0}}, \hat{x}_{j_{0}}^{\circ}\right)+\mathbb{E}_{\mathbf{x}_{j_{0}+1}}\left[J_{j_{0}+1}^{*}\left(\mathbf{x}_{j_{0}+1}, \hat{x}_{j_{0}+1: N}^{\circ}\right) \mid \mathbf{x}_{j_{0}}=x_{j_{0}}\right]<c_{j_{0}}^{\prime}\right\}
$$

is non-empty, where $J_{j_{0}+1}^{*}$ is defined in (18). Note that by Proposition III.13

$$
d^{2}\left(x_{j_{0}}, \hat{x}_{j_{0}}^{\circ}\right)+\mathbb{E}_{\mathbf{x}_{j_{0}+1}}\left[J_{j_{0}+1}^{*}\left(\mathbf{x}_{j_{0}+1}, \hat{x}_{j_{0}+1: N}^{\circ}\right) \mid \mathbf{x}_{j_{0}}=x_{j_{0}}\right]
$$

is a continuous function of $x_{j_{0}}$. Hence, for a positive constant $\epsilon$, the set defined by

$$
\mathbb{B}=\left\{x_{j_{0}} \in \mathbb{R}^{2} \times[0,2 \pi) \mid d^{2}\left(x_{j_{0}}, \hat{x}_{j_{0}}^{\circ}\right)+\mathbb{E}_{\mathbf{x}_{j_{0}+1}}\left[J_{j_{0}+1}^{*}\left(\mathbf{x}_{j_{0}+1}, \hat{x}_{j_{0}+1: N}^{\circ}\right) \mid \mathbf{x}_{j_{0}}=x_{j_{0}}\right]<c_{j_{0}}^{\prime}-\epsilon\right\}
$$

is non-empty and open.

For each $j$ in $\left\{j_{0}, \cdots, N\right\}$, let us define a function $\mathcal{P}_{j}^{\circ}: \mathbb{R}^{2} \times[0,2 \pi) \rightarrow\{0,1\}$ as follows:

$$
\mathcal{P}_{j}^{\circ}\left(x_{j}\right)= \begin{cases}0 & \text { if } x_{j} \in \underline{\mathbb{D}}_{j}^{\circ} \\ 1 & \text { otherwise }\end{cases}
$$

where

$$
\underline{\mathbb{D}}_{j}^{\circ}=\left\{x_{j} \in \mathbb{R}^{2} \times[0,2 \pi) \mid d^{2}\left(x_{j}, \hat{x}_{j}^{\circ}\right)+\mathbb{E}_{\mathbf{x}_{j+1}}\left[J_{j+1}^{*}\left(\mathbf{x}_{j+1}, \hat{x}_{j+1: N}^{\circ}\right) \mid \mathbf{x}_{j}=x_{j}\right]<c_{j}^{\prime}\right\}
$$

Also, let us select sequences of policies $\left\{\mathcal{P}_{k: N}^{(i)}\right\}_{i \in \mathbb{N}}$ and estimates $\left\{\hat{x}_{k: N}^{\prime(i)}\right\}_{i \in \mathbb{N}}$ as follows: for each $i$ in $\mathbb{N}$ and $j$ in $\{k, \cdots, N\}$,

$$
\begin{aligned}
& \mathcal{P}_{j}^{(i)}= \begin{cases}\mathcal{P}_{j}^{(i)} & \text { if } j \in\left\{k, \cdots, j_{0}-1\right\} \\
\mathcal{P}_{j}^{\circ} & \text { if } j \in\left\{j_{0}, \cdots, N\right\}\end{cases} \\
& \hat{x}_{j}^{(i)}= \begin{cases}\hat{x}_{j}^{(i)} & \text { if } j \in\left\{k, \cdots, j_{0}-1\right\} \\
\hat{x}_{j}^{\circ} & \text { if } j \in\left\{j_{0}, \cdots, N\right\}\end{cases}
\end{aligned}
$$

\footnotetext{
${ }^{15}$ Such $j_{0}$ always exists; otherwise according to Lemma F.15 the sequence $\left\{\hat{x}_{k: N}^{(i)}\right\}_{i \in \mathbb{N}}$ is bounded, which violates (H1)
} 
We argue that $\hat{x}_{k: N}^{(i-1)} \in \mathbb{K}_{i-1}$ and $\mathcal{G}\left(\hat{x}_{k: N}^{\prime(i-1)}\right)<\mathcal{G}\left(\hat{x}_{k: N}^{(i-1)}\right)$ hold for sufficiently large $i$. This contradicts the hypothesis (H2) hence it completes the proof of the lemma. In what follows, we show that this argument is valid. Note that by Lemma F.15. the sequence $\left\{\hat{x}_{j_{0}-1}^{(i-1)}\right\}_{i \in \mathbb{N}}$ is bounded. By Proposition D.11 and by the fact that $\mathcal{P}_{j}^{(i)}=\mathcal{P}_{j}^{(i)}$ for all $i$ in $\mathbb{N}$ and $j$ in $\left\{k, \cdots, j_{0}-1\right\}$, there exists a compact set $\mathbb{K}_{j_{0}-1}$ for which the following holds for all $i$ in $\mathbb{N}$ :

$$
\begin{aligned}
& \mathbb{P}\left(\mathbf{x}_{j_{0}-1} \in \mathbb{K}_{j_{0}-1} \mid \mathbf{R}_{k}^{\prime(i)}=0, \cdots, \mathbf{R}_{j_{0}-1}^{\prime(i)}=0\right) \\
& =\mathbb{P}\left(\mathbf{x}_{j_{0}-1} \in \mathbb{K}_{j_{0}-1} \mid \mathbf{R}_{k}^{(i)}=0, \cdots, \mathbf{R}_{j_{0}-1}^{(i)}=0\right)=1
\end{aligned}
$$

By Proposition D.12, we can write that

$$
\begin{aligned}
& \mathbb{P}\left(\mathbf{x}_{j_{0}} \in \mathbb{B} \mid \mathbf{R}_{k}^{(i)}=0, \cdots, \mathbf{R}_{j_{0}-1}^{\prime(i)}=0\right) \\
& =\int_{\mathbb{K}_{j_{0}-1}} \mathbb{P}\left(\mathbf{x}_{j_{0}} \in \mathbb{B} \mid \mathbf{x}_{j_{0}-1}=x\right) \mathrm{d} \mu_{j_{0}-1 \mid j_{0}-1}^{\prime(i)}
\end{aligned}
$$

where the probability measure $\mu_{j_{0}-1 \mid j_{0}-1}^{\prime(i)}$ is defined as follows: For each $\mathbb{A}$ in $\mathfrak{B}$,

$$
\mu_{j_{0}-1 \mid j_{0}-1}^{\prime(i)}(\mathbb{A})=\mathbb{P}\left(\mathbf{x}_{j_{0}-1} \in \mathbb{A} \mid \mathbf{R}_{k}^{\prime(i)}=0, \cdots, \mathbf{R}_{j_{0}-1}^{(i)}=0\right)
$$

Due to Assumption II.4 and the compactness of $\mathbb{K}_{j_{0}-1}$, for a positive constant $\delta_{j_{0}}$ and for the set $\mathbb{B}$ given by 66, the following holds for all $i$ in $\mathbb{N}$ :

$$
\begin{aligned}
& \mathbb{P}\left(\mathbf{x}_{j_{0}} \in \mathbb{B} \mid \mathbf{R}_{k}^{\prime(i)}=0, \cdots, \mathbf{R}_{j_{0}-1}^{(i)}=0\right) \\
& \geq \delta_{j_{0}} \cdot \mu_{j_{0}-1 \mid j_{0}-1}^{\prime(i)}\left(\mathbb{K}_{j_{0}-1}\right)=\delta_{j_{0}}
\end{aligned}
$$

Since the sequence $\left\{\hat{x}_{j}^{(i-1)}\right\}_{i \in \mathbb{N}}$ is bounded for all $j$ in $\left\{k, \cdots, j_{0}-1\right\}$, for sufficiently large $i$, we can see that $\hat{x}_{k: N}^{\prime(i-1)} \in \mathbb{K}_{i-1}$. In addition, by [14], 66, 67, and 687, we can see that the following relations hold for all $i$ in $\mathbb{N}$ :

$$
\begin{aligned}
& \mathbb{E}_{\mathbf{x}_{j_{0}}}\left[J_{j_{0}}\left(\mathbf{x}_{j_{0}}, \mathcal{P}_{j_{0}: N}^{\prime(i)}, \hat{x}_{j_{0}: N}^{\prime(i-1)}\right) \mid \mathbf{R}_{k}^{\prime(i)}=0, \cdots, \mathbf{R}_{j_{0}-1}^{\prime(i)}=0\right] \\
& =\left(\mathbb{E}_{\mathbf{x}_{j_{0}}}\left[d^{2}\left(\mathbf{x}_{j_{0}}, \hat{x}_{j_{0}}^{\circ}\right) \mid \mathbf{R}_{k}^{\prime(i)}=0, \cdots, \mathbf{R}_{j_{0}}^{\prime(i)}=0\right]+\mathbb{E}_{\mathbf{x}_{j_{0}+1}}\left[J_{j_{0}+1}^{*}\left(\mathbf{x}_{j_{0}+1}, \hat{x}_{j_{0}+1: N}^{\circ}\right) \mid \mathbf{R}_{k}^{\prime(i)}=0, \cdots, \mathbf{R}_{j_{0}}^{(i)}=0\right]\right) \\
& \quad \cdot \mathbb{P}\left(\mathbf{R}_{j_{0}}^{\prime(i)}=0 \mid \mathbf{R}_{k}^{\prime(i)}=0, \cdots, \mathbf{R}_{j_{0}-1}^{\prime(i)}=0\right)+c_{j_{0}}^{\prime} \cdot\left(1-\mathbb{P}\left(\mathbf{R}_{j_{0}}^{\prime(i)}=0 \mid \mathbf{R}_{k}^{\prime(i)}=0, \cdots, \mathbf{R}_{j_{0}-1}^{\prime(i)}=0\right)\right) \\
& \stackrel{(\mathbf{i})}{\leq}\left(\mathbb{E}_{\mathbf{x}_{j_{0}}}\left[d^{2}\left(\mathbf{x}_{j_{0}}, \hat{x}_{j_{0}}^{\circ}\right) \mid \mathbf{R}_{k}^{\prime(i)}=0, \cdots, \mathbf{R}_{j_{0}-1}^{\prime(i)}=0, \mathbf{x}_{j_{0}} \in \mathbb{B}\right]\right. \\
& \left.\quad+\mathbb{E}_{\mathbf{x}_{j_{0}+1}}\left[J_{j_{0}+1}^{*}\left(\mathbf{x}_{j_{0}+1}, \hat{x}_{j_{0}+1: N}^{o}\right) \mid \mathbf{R}_{k}^{\prime(i)}=0, \cdots, \mathbf{R}_{j_{0}-1}^{\prime(i)}=0, \mathbf{x}_{j_{0}} \in \mathbb{B}\right]\right) \\
& \quad \cdot \mathbb{P}\left(\mathbf{x}_{j_{0}} \in \mathbb{B} \mid \mathbf{R}_{k}^{\prime(i)}=0, \cdots, \mathbf{R}_{j_{0}-1}^{\prime(i)}=0\right)+c_{j_{0}}^{\prime} \cdot\left(1-\mathbb{P}\left(\mathbf{x}_{j_{0}} \in \mathbb{B} \mid \mathbf{R}_{k}^{\prime(i)}=0, \cdots, \mathbf{R}_{j_{0}-1}^{\prime(i)}=0\right)\right) \\
& \stackrel{(i \mathbf{i})}{<}\left(c_{j_{0}}^{\prime}-\epsilon\right) \cdot \mathbb{P}\left(\mathbf{x}_{j_{0}} \in \mathbb{B} \mid \mathbf{R}_{k}^{\prime(i)}=0, \cdots, \mathbf{R}_{j_{0}-1}^{\prime(i)}=0\right)+c_{j_{0}}^{\prime} \cdot\left(1-\mathbb{P}\left(\mathbf{x}_{j_{0}} \in \mathbb{B} \mid \mathbf{R}_{k}^{\prime(i)}=0, \cdots, \mathbf{R}_{j_{0}-1}^{\prime(i)}=0\right)\right) \\
& \stackrel{(\mathrm{iiii})}{\leq} c_{j_{0}}^{\prime}-\epsilon \cdot \delta_{j_{0}}
\end{aligned}
$$


To obtain (i), we use the fact that

$$
d^{2}\left(\mathbf{x}_{j_{0}}, \hat{x}_{j_{0}}^{\circ}\right)+\mathbb{E}_{\mathbf{x}_{j_{0}+1}}\left[J_{j_{0}+1}^{*}\left(\mathbf{x}_{j_{0}+1}, \hat{x}_{j_{0}+1: N}^{\circ}\right) \mid \mathbf{x}_{j_{0}}\right]<c_{j_{0}}^{\prime}
$$

if $\mathbf{R}_{j_{0}}^{(i)}=0$ (or equivalently $\mathbf{x}_{j_{0}} \in \underline{\mathbb{D}}_{j_{0}}^{\circ}$ ), and $\mathbb{B}$ is a subset of $\underline{\mathbb{D}}_{j_{0}}^{\circ}$; whereas (ii) and (iii) follow from 66 and (68), respectively. By a similar argument as in the proof of Proposition [II.14, from 664) and 696, we can observe that for sufficiently large $i$, there exists $\hat{x}_{k: N}^{(i-1)}$ in $\mathbb{K}_{i-1}$ for which it holds that

$$
\mathcal{G}\left(\hat{x}_{k: N}^{(i-1)}\right) \leq \mathbb{E}_{\mathbf{x}_{k}}\left[J_{k}\left(\mathbf{x}_{k}, \mathcal{P}_{k: N}^{(i)}, \hat{x}_{k: N}^{(i-1)}\right)\right]<\mathbb{E}_{\mathbf{x}_{k}}\left[J_{k}\left(\mathbf{x}_{k}, \mathcal{P}_{k: N}^{(i)}, \hat{x}_{k: N}^{(i-1)}\right)\right]=\mathcal{G}\left(\hat{x}_{k: N}^{(i-1)}\right)
$$

\section{G. Proofs of Lemmas III.23 and III.24}

Proof of Lemma III.23. To prove the lemma, we first claim that the following hold for all $j$ in $\{k, \cdots, N\}$ :

$$
\begin{aligned}
\lim _{l \rightarrow \infty} \mathbb{E}\left[\mathbf{p}_{1, j} \mid \mathbf{R}_{k}^{\left(i_{l}\right)}=0, \cdots, \mathbf{R}_{j}^{\left(i_{l}\right)}=0\right] & =\mathbb{E}\left[\mathbf{p}_{1, j} \mid \mathbf{R}_{k}=0, \cdots, \mathbf{R}_{j}=0\right] \\
\lim _{l \rightarrow \infty} \mathbb{E}\left[\mathbf{p}_{2, j} \mid \mathbf{R}_{k}^{\left(i_{l}\right)}=0, \cdots, \mathbf{R}_{j}^{\left(i_{l}\right)}=0\right] & =\mathbb{E}\left[\mathbf{p}_{2, j} \mid \mathbf{R}_{k}=0, \cdots, \mathbf{R}_{j}=0\right] \\
\lim _{l \rightarrow \infty} \mathbb{E}\left[\sin \boldsymbol{\theta}_{j} \mid \mathbf{R}_{k}^{\left(i_{l}\right)}=0, \cdots, \mathbf{R}_{j}^{\left(i_{l}\right)}=0\right] & =\mathbb{E}\left[\sin \boldsymbol{\theta}_{j} \mid \mathbf{R}_{k}=0, \cdots, \mathbf{R}_{j}=0\right] \\
\lim _{l \rightarrow \infty} \mathbb{E}\left[\cos \boldsymbol{\theta}_{j} \mid \mathbf{R}_{k}^{\left(i_{l}\right)}=0, \cdots, \mathbf{R}_{j}^{\left(i_{l}\right)}=0\right] & =\mathbb{E}\left[\cos \boldsymbol{\theta}_{j} \mid \mathbf{R}_{k}=0, \cdots, \mathbf{R}_{j}=0\right]
\end{aligned}
$$

subject to $\mathbf{R}_{j}^{\left(i_{l}\right)}=\mathcal{P}_{j}^{\left(i_{l}\right)}\left(\mathbf{x}_{j}\right)$ and $\mathbf{R}_{j}=\mathcal{P}_{j}\left(\mathbf{x}_{j}\right)$ for each $l$ in $\mathbb{N}$ and $j$ in $\{k, \cdots, N\}$.

For notational convenient, let us define

$$
\alpha=\mathbb{E}^{2}\left[\sin \boldsymbol{\theta}_{j} \mid \mathbf{R}_{k}=0, \cdots, \mathbf{R}_{j}=0\right]+\mathbb{E}^{2}\left[\cos \boldsymbol{\theta}_{j} \mid \mathbf{R}_{k}=0, \cdots, \mathbf{R}_{j}=0\right]
$$

If $\alpha$ is non-zero, then by Corollary III.12, $\hat{x}_{j}^{\left(i_{l}\right)}=\left(\begin{array}{llll}\hat{p}_{1, j}^{\left(i_{l}\right)} & \hat{p}_{2, j}^{\left(i_{l}\right)} & \hat{\theta}_{j}^{\left(i_{l}\right)}\end{array}\right)^{T}$ and $\hat{x}_{j}=\left(\begin{array}{lll}\hat{p}_{1, j} & \hat{p}_{2, j} & \hat{\theta}_{j}\end{array}\right)^{T}$ satisfy

$$
\begin{aligned}
p_{1, j}^{\left(i_{l}\right)} & =\mathbb{E}\left[\mathbf{p}_{1, j} \mid \mathbf{R}_{k}^{\left(i_{l}\right)}=0, \cdots, \mathbf{R}_{j}^{\left(i_{l}\right)}=0\right] \\
p_{2, j}^{\left(i_{l}\right)} & =\mathbb{E}\left[\mathbf{p}_{2, j} \mid \mathbf{R}_{k}^{\left(i_{l}\right)}=0, \cdots, \mathbf{R}_{j}^{\left(i_{l}\right)}=0\right] \\
\sin \hat{\theta}_{j}^{\left(i_{l}\right)} & =\frac{\mathbb{E}\left[\sin \boldsymbol{\theta}_{j} \mid \mathbf{R}_{k}^{\left(i_{l}\right)}=0, \cdots, \mathbf{R}_{j}^{\left(i_{l}\right)}=0\right]}{\mathbb{E}^{2}\left[\sin \boldsymbol{\theta}_{j} \mid \mathbf{R}_{k}^{\left(i_{l}\right)}=0, \cdots, \mathbf{R}_{j}^{\left(i_{l}\right)}=0\right]+\mathbb{E}^{2}\left[\cos \boldsymbol{\theta}_{j} \mid \mathbf{R}_{k}^{\left(i_{l}\right)}=0, \cdots, \mathbf{R}_{j}^{\left(i_{l}\right)}=0\right]} \\
\cos \hat{\theta}_{j}^{\left(i_{l}\right)} & =\frac{\mathbb{E}\left[\cos \boldsymbol{\theta}_{j} \mid \mathbf{R}_{k}^{\left(i_{l}\right)}=0, \cdots, \mathbf{R}_{j}^{\left(i_{l}\right)}=0\right]}{\mathbb{E}^{2}\left[\sin \boldsymbol{\theta}_{j} \mid \mathbf{R}_{k}^{\left(i_{l}\right)}=0, \cdots, \mathbf{R}_{j}^{\left(i_{l}\right)}=0\right]+\mathbb{E}^{2}\left[\cos \boldsymbol{\theta}_{j} \mid \mathbf{R}_{k}^{\left(i_{l}\right)}=0, \cdots, \mathbf{R}_{j}^{\left(i_{l}\right)}=0\right]}
\end{aligned}
$$

and

$$
\lim _{l \rightarrow \infty} d\left(\hat{x}_{j}^{\left(i_{l}\right)}, \hat{x}_{j}\right)=0
$$


For each $j$ in $\{k, \cdots, N\}$, let us define $\hat{x}_{j}^{*}=\left(\begin{array}{lll}\hat{p}_{1, j}^{*} \quad \hat{p}_{2, j}^{*} \quad \hat{\theta}_{j}^{*}\end{array}\right)$ as

$$
\begin{aligned}
p_{1, j}^{*} & =\mathbb{E}\left[\mathbf{p}_{1, j} \mid \mathbf{R}_{k}=0, \cdots, \mathbf{R}_{j}=0\right] \\
p_{2, j}^{*} & =\mathbb{E}\left[\mathbf{p}_{2, j} \mid \mathbf{R}_{k}=0, \cdots, \mathbf{R}_{j}=0\right] \\
\sin \hat{\theta}_{j}^{*} & =\alpha^{-1} \cdot \mathbb{E}\left[\sin \boldsymbol{\theta}_{j} \mid \mathbf{R}_{k}=0, \cdots, \mathbf{R}_{j}=0\right] \\
\cos \hat{\theta}_{j}^{*} & =\alpha^{-1} \cdot \mathbb{E}\left[\cos \boldsymbol{\theta}_{j} \mid \mathbf{R}_{k}=0, \cdots, \mathbf{R}_{j}=0\right]
\end{aligned}
$$

Note that by Corollary III.12, it holds that $\hat{x}_{k: N}^{*} \in \mathfrak{X}\left(\mathcal{P}_{k: N}\right)$. From (70) and 71], we can observe that

$$
d\left(\hat{x}_{j}, \hat{x}_{j}^{*}\right) \leq d\left(\hat{x}_{j}^{\left(i_{l}\right)}, \hat{x}_{j}^{*}\right)+d\left(\hat{x}_{j}^{\left(i_{l}\right)}, \hat{x}_{j}\right) \stackrel{l \rightarrow \infty}{\longrightarrow} 0
$$

Therefore, we conclude that $\hat{x}_{k: N} \in \mathfrak{X}\left(\mathcal{P}_{k: N}\right)$.

Otherwise, if $\alpha$ is zero then the value of

$$
\mathbb{E}_{\mathbf{x}_{j}}\left[d^{2}\left(\mathbf{x}_{j}, \hat{x}_{j}\right) \mid \mathbf{R}_{k}=0, \cdots, \mathbf{R}_{j}=0\right]
$$

does not depend on $\hat{\theta}_{j}$, and by Proposition III.11 and Corollary III.12, we can show that $\hat{x}_{k: N} \in \mathfrak{X}\left(\mathcal{P}_{k: N}\right)$ if the following hold for all $j$ in $\{k, \cdots, N\}$ :

$$
\begin{aligned}
& p_{1, j}=\mathbb{E}\left[\mathbf{p}_{1, j} \mid \mathbf{R}_{k}=0, \cdots, \mathbf{R}_{j}=0\right] \\
& p_{2, j}=\mathbb{E}\left[\mathbf{p}_{2, j} \mid \mathbf{R}_{k}=0, \cdots, \mathbf{R}_{j}=0\right]
\end{aligned}
$$

This can be verified by similar arguments given above and 70 . It remains to prove the claim.

Proof of the Claim: Notice that $\mathbf{p}_{1, j}, \mathbf{p}_{2, j}, \sin \hat{\boldsymbol{\theta}}_{j}, \cos \hat{\boldsymbol{\theta}}_{j}$ are all continuous functions of $\mathbf{x}_{j}$. Hence to show that (70) is true, it is sufficient to show that the following holds for any continuous function $g: \mathbb{R}^{2} \times[0,2 \pi) \rightarrow \mathbb{R}$ :

$$
\lim _{l \rightarrow \infty} \mathbb{E}\left[g\left(\mathbf{x}_{j}\right) \mid \mathbf{R}_{k}^{\left(i_{l}\right)}=0, \cdots, \mathbf{R}_{j}^{\left(i_{l}\right)}=0\right]=\mathbb{E}\left[g\left(\mathbf{x}_{j}\right) \mid \mathbf{R}_{k}=0, \cdots, \mathbf{R}_{j}=0\right]
$$

Recall the definitions of $\mu_{j \mid j}^{\left(i_{l}\right)}$ and $\mu_{j \mid j}$ given in 25. Since $\left\{\mathcal{P}_{k: N}^{\left(i_{l}\right)}\right\}_{l \in \mathbb{N}}$ converges to $\mathcal{P}_{k: N}$, by Definition III.17. it holds that $\mu_{j \mid j}^{\left(i_{l}\right)} \rightarrow \mu_{j \mid j}$ for all $j$ in $\{k, \cdots, N\}$. Since $\left(\mathbb{R}^{2} \times[0,2 \pi), d\right)$ is a complete, separable metric space, by the convergence of $\left\{\mu_{j \mid j}^{\left(i_{l}\right)}\right\}_{i \in \mathbb{N}}$ and the Skorokhod representation theorem [13], there exist a sequence of random variables $\left\{\mathbf{y}_{j}^{\left(i_{l}\right)}\right\}_{l \in \mathbb{N}}$ and a random variable $\mathbf{y}_{j}$ all defined on a common probability space $(\Omega, \mathfrak{F}, \nu)$ in which the following three facts are true:

(F1) $\mu_{j \mid j}^{\left(i_{l}\right)}$ is the probability measure of $\mathbf{y}_{j}^{\left(i_{l}\right)}$, i.e., $\nu\left(\left\{\omega \in \Omega \mid \mathbf{y}_{j}^{\left(i_{l}\right)}(\omega) \in \mathbb{A}\right\}\right)=\mu_{j \mid j}^{\left(i_{l}\right)}(\mathbb{A})$ for each $\mathbb{A}$ in $\mathfrak{B}$.

(F2) $\mu_{j \mid j}$ is the probability measure of $\mathbf{y}_{j}$, i.e., $\nu\left(\left\{\omega \in \Omega \mid \mathbf{y}_{j}(\omega) \in \mathbb{A}\right\}\right)=\mu_{j \mid j}(\mathbb{A})$ for each $\mathbb{A}$ in $\mathfrak{B}$.

(F3) $\left\{\mathbf{y}_{j}^{\left(i_{l}\right)}\right\}_{i \in \mathbb{N}}$ converges to $\mathbf{y}_{j}$ almost surely.

Since $\left\{\hat{x}_{j}^{\left(i_{l}-1\right)}\right\}_{l \in \mathbb{N}}$ is a convergent sequence, according to Proposition D.11 there is a compact set $\mathbb{K}_{j}$ for which $\mu_{j \mid j}^{\left(i_{l}\right)}\left(\mathbb{K}_{j}\right)=1$ for all $l$ in $\mathbb{N}$. Hence, by $(\mathbf{F 1})$, the following holds for a positive real $\beta$ :

$$
\int\left\{\omega \in \Omega|| g\left(\mathbf{y}_{j}^{\left(i_{l}\right)}(\omega)\right) \mid>\beta\right\}\left|g\left(\mathbf{y}_{j}^{\left(i_{l}\right)}(\omega)\right)\right| \mathrm{d} \nu=\int_{\left\{x \in \mathbb{R}^{2} \times[0,2 \pi)|| g(x) \mid>\beta\right\}}|g(x)| \mathrm{d} \mu_{j \mid j}^{\left(i_{l}\right)}=0
$$


for all $l$ in $\mathbb{N}$. In conjunction with $[\mathbf{F 3}$ ) by an application of Theorem 10.3.6 in [10], we have that

$$
\lim _{l \rightarrow \infty} \int_{\Omega} g\left(\mathbf{y}_{j}^{\left(i_{l}\right)}(\omega)\right) \mathrm{d} \nu=\int_{\Omega} g\left(\mathbf{y}_{j}(\omega)\right) \mathrm{d} \nu
$$

Therefore, from (F2) and (74), we can see that

$$
\begin{aligned}
\lim _{l \rightarrow \infty} \int_{\mathbb{R}^{2} \times[0,2 \pi)} g(x) \mathrm{d} \mu_{j \mid j}^{\left(i_{l}\right)} & =\lim _{l \rightarrow \infty} \int_{\Omega} g\left(\mathbf{y}_{j}^{\left(i_{l}\right)}(\omega)\right) \mathrm{d} \nu \\
& =\int_{\Omega} g\left(\mathbf{y}_{j}(\omega)\right) \mathrm{d} \nu \\
& =\int_{\mathbb{R}^{2} \times[0,2 \pi)} g(x) \mathrm{d} \mu_{j \mid j}
\end{aligned}
$$

This proves the claim.

Lemma G.16: Let $\left\{\hat{x}_{k: N}^{(i)}\right\}_{i \in \mathbb{N}}$ be a sequence of estimates that converges to $\hat{x}_{k: N}$. The following inclusions hold for all $j$ in $\{k, \cdots, N\}$ :

$$
\begin{aligned}
& \overline{\mathbb{D}}_{j} \supset \bigcap_{i \in \mathbb{N}} \bigcup_{l \geq i} \overline{\mathbb{D}}_{j}^{(l)} \\
& \underline{\mathbb{D}}_{j} \subset \bigcup_{i \in \mathbb{N}} \bigcap_{l \geq i} \mathbb{D}_{j}^{(l)}
\end{aligned}
$$

where

$$
\begin{aligned}
& \overline{\mathbb{D}}_{j}=\left\{x_{j} \in \mathbb{R}^{2} \times[0,2 \pi) \mid d^{2}\left(x_{j}, \hat{x}_{j}\right)+\mathbb{E}_{\mathbf{x}_{j+1}}\left[J_{j+1}^{*}\left(\mathbf{x}_{j+1}, \hat{x}_{j+1: N}\right) \mid \mathbf{x}_{j}=x_{j}\right] \leq c_{j}^{\prime}\right\} \\
& \underline{\mathbb{D}}_{j}=\left\{x_{j} \in \mathbb{R}^{2} \times[0,2 \pi) \mid d^{2}\left(x_{j}, \hat{x}_{j}\right)+\mathbb{E}_{\mathbf{x}_{j+1}}\left[J_{j+1}^{*}\left(\mathbf{x}_{j+1}, \hat{x}_{j+1: N}\right) \mid \mathbf{x}_{j}=x_{j}\right]<c_{j}^{\prime}\right\}
\end{aligned}
$$

and

$$
\begin{aligned}
& \overline{\mathbb{D}}_{j}^{(i)}=\left\{x_{j} \in \mathbb{R}^{2} \times[0,2 \pi) \mid d^{2}\left(x_{j}, \hat{x}_{j}^{(i)}\right)+\mathbb{E}_{\mathbf{x}_{j+1}}\left[J_{j+1}^{*}\left(\mathbf{x}_{j+1}, \hat{x}_{j+1: N}^{(i)}\right) \mid \mathbf{x}_{j}=x_{j}\right] \leq c_{j}^{\prime}\right\} \\
& \underline{\mathbb{D}}_{j}^{(i)}=\left\{x_{j} \in \mathbb{R}^{2} \times[0,2 \pi) \mid d^{2}\left(x_{j}, \hat{x}_{j}^{(i)}\right)+\mathbb{E}_{\mathbf{x}_{j+1}}\left[J_{j+1}^{*}\left(\mathbf{x}_{j+1}, \hat{x}_{j+1: N}^{(i)}\right) \mid \mathbf{x}_{j}=x_{j}\right]<c_{j}^{\prime}\right\}
\end{aligned}
$$

where $J_{j+1}^{*}$ is defined in 18 .

Proof: Let $x_{j}$ be an element of $\bigcap_{i \in \mathbb{N}} \bigcup_{l \geq i} \overline{\mathbb{D}}_{j}^{(l)}$. By definition, there exists an infinite index set $\left\{i_{l}\right\}_{l \in \mathbb{N}}$ for which $x_{j} \in \overline{\mathbb{D}}_{j}^{\left(i_{l}\right)}$ holds for all $l$ in $\mathbb{N}$. Hence, we can see that

$$
d^{2}\left(x_{j}, \hat{x}_{j}^{\left(i_{l}\right)}\right)+\mathbb{E}_{\mathbf{x}_{j+1}}\left[J_{j+1}^{*}\left(\mathbf{x}_{j+1}, \hat{x}_{j+1: N}^{\left(i_{l}\right)}\right) \mid \mathbf{x}_{j}=x_{j}\right] \leq c_{j}^{\prime}
$$

holds for all $l$ in $\mathbb{N}$. Using Proposition III.13 and by the fact that $\left\{\hat{x}_{k: N}^{(i)}\right\}_{i \in \mathbb{N}}$ converges to $\hat{x}_{k: N}$, we can derive

$$
d^{2}\left(x_{j}, \hat{x}_{j}\right)+\mathbb{E}_{\mathbf{x}_{j+1}}\left[J_{j+1}^{*}\left(\mathbf{x}_{j+1}, \hat{x}_{j+1: N}\right) \mid \mathbf{x}_{j}=x_{j}\right] \leq c_{j}^{\prime}
$$

which shows that $x_{j} \in \overline{\mathbb{D}}_{j}$. This proves 76a).

To show that $76 \mathrm{~b}$ is true, we consider

$$
\underline{\mathbb{D}}_{j}^{c} \supset \bigcap_{i \in \mathbb{N}} \bigcup_{l \geq i}\left(\underline{\mathbb{D}}_{j}^{(l)}\right)^{c}
$$

As the rest of the proof is similar to the above arguments, we omit the detail for brevity. 
Proof of Lemma $I I I .24$. For every $\mathbb{A}$ in $\mathfrak{B}$, let us define

$$
\begin{gathered}
\mu_{j \mid j-1}^{(i)}(\mathbb{A})=\mathbb{P}\left(\mathbf{x}_{j} \in \mathbb{A} \mid \mathbf{R}_{k}^{(i)}=0, \cdots, \mathbf{R}_{j-1}^{(i)}=0\right) \\
\mu_{j \mid j}^{(i)}(\mathbb{A})=\mathbb{P}\left(\mathbf{x}_{j} \in \mathbb{A} \mid \mathbf{R}_{k}^{(i)}=0, \cdots, \mathbf{R}_{j}^{(i)}=0\right)
\end{gathered}
$$

subject to $\mathbf{R}_{j}^{(i)}=\mathcal{P}_{j}^{(i)}\left(\mathbf{x}_{j}\right)$ for all $i$ in $\mathbb{N}$ and $j$ in $\{k, \cdots, N\}$. Since $\left\{\hat{x}_{k: N}^{\left(i_{l}-1\right)}\right\}_{l \in \mathbb{N}}$ is a convergent sequence, according to Proposition D.11 there exist compact subsets $\left\{\mathbb{K}_{j}\right\}_{j=k}^{N}$ for which the following holds for all $l$ in $\mathbb{N}$ and $j$ in $\{k, \cdots, N\}$ :

$$
\mu_{j \mid j}^{\left(i_{l}\right)}\left(\mathbb{K}_{j}\right)=1
$$

Hence, for all $j$ in $\{k, \cdots, N\}$, the probability measures $\left\{\mu_{j \mid j}^{\left(i_{l}\right)}\right\}_{l \in \mathbb{N}}$ are uniformly tight in the sense of Definition C.7 By Theorem 11.5.4 in [10], for each $j$ in $\{k, \cdots, N\}$, there exists a subsequence $\left\{\mu_{j \mid j}^{\left(i_{l}^{\prime}\right)}\right\}_{l \in \mathbb{N}}$ that converges to a probability measure $\mu_{j \mid j}$ defined on $\left(\mathbb{R}^{2} \times[0,2 \pi), \mathfrak{B}\right)$. In addition, note that $\mathbb{P}\left(\mathbf{R}_{j}^{(i)}=0 \mid \mathbf{R}_{k}^{(i)}=0, \cdots, \mathbf{R}_{j-1}^{(i)}\right)$ takes a value in a compact set $[\epsilon, 1]$ for all $i$ in $\mathbb{N}$ and $j$ in $\{k, \cdots, N\}$. Hence, there is an infinite index set $\left\{i_{l}^{\prime \prime}\right\}_{l \in \mathbb{N}}$ of $\left\{i_{l}^{\prime}\right\}_{l \in \mathbb{N}}$ for which the following holds for all $j$ in $\{k, \cdots, N\}$ :

$$
\lim _{l \rightarrow \infty} \mathbb{P}\left(\mathbf{R}_{j}^{\left(i_{l}^{\prime \prime}\right)}=0 \mid \mathbf{R}_{k}^{\left(i_{l}^{\prime \prime}\right)}=0, \cdots, \mathbf{R}_{j-1}^{\left(i_{l}^{\prime \prime}\right)}=0\right)=q_{j}
$$

where $q_{j}$ belongs to $[\epsilon, 1]$. For clear and simple presentation, without loss of generality, we prove the lemma by imposing the following three assumptions: For each $j$ in $\{k, \cdots, N\}$,

(A1) The estimates $\left\{\hat{x}_{k: N}^{(i-1)}\right\}_{i \in \mathbb{N}}$ converge to $\hat{x}_{k: N}^{\prime}$.

(A2) The probability measures $\left\{\mu_{j \mid j}^{(i)}\right\}_{i \in \mathbb{N}}$ converge to $\mu_{j \mid j}$.

(A3) $\lim _{i \rightarrow \infty} \mathbb{P}\left(\mathbf{R}_{j}^{(i)}=0 \mid \mathbf{R}_{k}^{(i)}=0, \cdots, \mathbf{R}_{j-1}^{(i)}=0\right)=q_{j}$ holds, where $q_{j}$ belongs to $[\epsilon, 1]$

To complete the proof of the lemma, it is sufficient to show that there exist policies $\mathcal{P}_{k: N}$ for which

(F1) For every $\mathbb{A}$ in $\mathfrak{B}$, it holds that

$$
\mu_{j \mid j}(\mathbb{A})=\mathbb{P}\left(\mathbf{x}_{j} \in \mathbb{A} \mid \mathbf{R}_{k}=0, \cdots, \mathbf{R}_{j}=0\right)
$$

and

$$
q_{j}=\mathbb{P}\left(\mathbf{R}_{j}=0 \mid \mathbf{R}_{k}=0, \cdots, \mathbf{R}_{j-1}=0\right)
$$

subject to $\mathbf{R}_{j}=\mathcal{P}_{j}\left(\mathbf{x}_{j}\right)$ for all $j$ in $\{k, \cdots, N\}$.

(F2) The policies $\mathcal{P}_{k: N}$ belong to $\mathfrak{P}\left(\hat{x}_{k: N}^{\prime}\right)$, where $\hat{x}_{k: N}^{\prime}$ is the limit of $\left\{\hat{x}_{k: N}^{(i-1)}\right\}_{i \in \mathbb{N}}$.

For notational convenience, let us define

$$
\begin{aligned}
& \overline{\mathbb{D}}_{j}=\left\{x_{j} \in \mathbb{R}^{2} \times[0,2 \pi) \mid d^{2}\left(x_{j}, \hat{x}_{j}^{\prime}\right)+\mathbb{E}_{\mathbf{x}_{j+1}}\left[J_{j+1}^{*}\left(\mathbf{x}_{j+1}, \hat{x}_{j+1: N}^{\prime}\right) \mid \mathbf{x}_{j}=x_{j}\right] \leq c_{j}^{\prime}\right\} \\
& \underline{\mathbb{D}}_{j}=\left\{x_{j} \in \mathbb{R}^{2} \times[0,2 \pi) \mid d^{2}\left(x_{j}, \hat{x}_{j}^{\prime}\right)+\mathbb{E}_{\mathbf{x}_{j+1}}\left[J_{j+1}^{*}\left(\mathbf{x}_{j+1}, \hat{x}_{j+1: N}^{\prime}\right) \mid \mathbf{x}_{j}=x_{j}\right]<c_{j}^{\prime}\right\}
\end{aligned}
$$


and

$$
\begin{aligned}
& \overline{\mathbb{D}}_{j}^{(i)}=\left\{x_{j} \in \mathbb{R}^{2} \times[0,2 \pi) \mid d^{2}\left(x_{j}, \hat{x}_{j}^{(i-1)}\right)+\mathbb{E}_{\mathbf{x}_{j+1}}\left[J_{j+1}^{*}\left(\mathbf{x}_{j+1}, \hat{x}_{j+1: N}^{(i-1)}\right) \mid \mathbf{x}_{j}=x_{j}\right] \leq c_{j}^{\prime}\right\} \\
& \underline{\mathbb{D}}_{j}^{(i)}=\left\{x_{j} \in \mathbb{R}^{2} \times[0,2 \pi) \mid d^{2}\left(x_{j}, \hat{x}_{j}^{(i-1)}\right)+\mathbb{E}_{\mathbf{x}_{j+1}}\left[J_{j+1}^{*}\left(\mathbf{x}_{j+1}, \hat{x}_{j+1: N}^{(i-1)}\right) \mid \mathbf{x}_{j}=x_{j}\right]<c_{j}^{\prime}\right\}
\end{aligned}
$$

for each $i$ in $\mathbb{N}$ and $j$ in $\{k, \cdots, N\}$, where $J_{j+1}^{*}$ is defined in (18). Note that according to Proposition III.13, the sets $\overline{\mathbb{D}}_{j}$ and $\overline{\mathbb{D}}_{j}^{(i)}$ are closed, and the sets $\underline{\mathbb{D}}_{j}$ and $\underline{\mathbb{D}}_{j}^{(i)}$ are open.

We first make the following two claims to show that (F1) is true.

Claim 1: For each $\mathbb{A}$ in $\mathfrak{B}$, let us define

$$
\mu_{j \mid j-1}(\mathbb{A}) \stackrel{\text { def }}{=} \int_{\mathbb{R}^{2} \times[0,2 \pi)} \mathbb{P}\left(\mathbf{x}_{j} \in \mathbb{A} \mid \mathbf{x}_{j-1}=x\right) \mathrm{d} \mu_{j-1 \mid j-1}
$$

Then, $\mu_{j \mid j-1}$ is a probability measure on $\left(\mathbb{R}^{2} \times[0,2 \pi), \mathfrak{B}\right)$, and it holds that

$$
\lim _{i \rightarrow \infty} \mu_{j \mid j-1}^{(i)}(\mathbb{A})=\mu_{j \mid j-1}(\mathbb{A})
$$

for all $\mathbb{A}$ in $\mathfrak{B}$.

To prove the claim, based on Proposition D.12, we note that

$$
\mu_{j \mid j-1}^{(i)}(\mathbb{A})=\int_{\mathbb{R}^{2} \times[0,2 \pi)} \mathbb{P}\left(\mathbf{x}_{j} \in \mathbb{A} \mid \mathbf{x}_{j-1}=x\right) \mathrm{d} \mu_{j-1 \mid j-1}^{(i)}
$$

holds for each $\mathbb{A}$ in $\mathfrak{B}$. By definition, for fixed $x$ in $\mathbb{R}^{2} \times[0,2 \pi)$, the mapping $\mathbb{A} \mapsto \mathbb{P}\left(\mathbf{x}_{j} \in \mathbb{A} \mid \mathbf{x}_{j-1}=x\right)$ defines a probability measure on $\left(\mathbb{R}^{2} \times[0,2 \pi), \mathfrak{B}\right)$. In conjunction with Assumption II.4. we can see that $x \mapsto \mathbb{P}\left(\mathbf{x}_{j} \in \mathbb{A} \mid \mathbf{x}_{j-1}=x\right)$ is a bounded, continuous function. Hence, using (A2), we have that

$$
\begin{aligned}
\lim _{i \rightarrow \infty} \mu_{j \mid j-1}^{(i)}(\mathbb{A}) & =\lim _{i \rightarrow \infty} \int_{\mathbb{R}^{2} \times[0,2 \pi)} \mathbb{P}\left(\mathbf{x}_{j} \in \mathbb{A} \mid \mathbf{x}_{j-1}=x\right) \mathrm{d} \mu_{j-1 \mid j-1}^{(i)} \\
& =\int_{\mathbb{R}^{2} \times[0,2 \pi)} \mathbb{P}\left(\mathbf{x}_{j} \in \mathbb{A} \mid \mathbf{x}_{j-1}=x\right) \mathrm{d} \mu_{j-1 \mid j-1}=\mu_{j \mid j-1}(\mathbb{A})
\end{aligned}
$$

Lastly, the argument that $\mu_{j \mid j-1}$ is a probability measure on $\left(\mathbb{R}^{2} \times[0,2 \pi), \mathfrak{B}\right)$ follows from $(86)$ and the fact that $\mathbb{A} \mapsto \mathbb{P}\left(\mathbf{x}_{j} \in \mathbb{A} \mid \mathbf{x}_{j-1}=x\right)$ is a probability measure on $\left(\mathbb{R}^{2} \times[0,2 \pi), \mathfrak{B}\right)$.

Claim 2: There exists a measurable function $f_{j}: \mathbb{R}^{2} \times[0,2 \pi) \rightarrow[0,1]$ for which

$$
\mu_{j \mid j}(\mathbb{A})=\frac{\int_{\mathbb{A}} f_{j} \mathrm{~d} \mu_{j \mid j-1}}{q_{j}}
$$

holds for all $\mathbb{A}$ in $\mathfrak{B}$, where $\mu_{j \mid j-1}$ is defined in 84 .

Based on Lemma C.8 and Proposition D.12, for any open set $\mathbb{O}$, we can see that the following relations hold:

$$
\begin{aligned}
\mu_{j \mid j}(\mathbb{O}) \leq \liminf _{i \rightarrow \infty} \mu_{j \mid j}^{(i)}(\mathbb{O}) & \stackrel{(\mathbf{i})}{\leq} \lim _{i \rightarrow \infty} \frac{\mu_{j \mid j-1}^{(i)}(\mathbb{O})}{\mathbb{P}\left(\mathbf{R}_{j}^{(i)}=0 \mid \mathbf{R}_{k}^{(i)}=0, \cdots, \mathbf{R}_{j-1}^{(i)}=0\right)} \\
& \stackrel{(i \mathbf{i})}{=} \frac{\mu_{j \mid j-1}(\mathbb{O})}{q_{j}}
\end{aligned}
$$


where (i) follows from Proposition D.12, and (ii) follows from Claim 1 and (A3). We argue that the following holds for any set $\mathbb{A}$ in $\mathfrak{B}$ :

$$
\mu_{j \mid j}(\mathbb{A}) \leq \frac{\mu_{j \mid j-1}(\mathbb{A})}{q_{j}}
$$

To justify the argument, by contradiction, suppose that for a set $\mathbb{A}$ in $\mathfrak{B}$ it holds that

$$
\mu_{j \mid j}(\mathbb{A})>\frac{\mu_{j \mid j-1}(\mathbb{A})}{q_{j}}
$$

By the closed regularity theorem (see Theorem 7.1.3 in [10]) and Remark C.5 we can choose an open set $\mathbb{O}$ containing $\mathbb{A}$ for which the following holds:

$$
\begin{aligned}
\mu_{j \mid j}(\mathbb{O}) \geq \mu_{j \mid j}(\mathbb{A}) & >\frac{\mu_{j \mid j-1}(\mathbb{O})}{q_{j}} \\
& \geq \frac{\mu_{j \mid j-1}(\mathbb{A})}{q_{j}}
\end{aligned}
$$

This contradicts 88 .

Notice that [89] implies that $\mu_{j \mid j}$ is absolutely continuous with respect to $\mu_{j \mid j-1}$. According to the RadonNikodym theorem, there is a measurable function $f_{j}: \mathbb{R}^{2} \times[0,2 \pi) \rightarrow \mathbb{R}_{+}$for which the following holds for all $\mathbb{A}$ in $\mathfrak{B}$ :

$$
\mu_{j \mid j}(\mathbb{A})=\frac{\int_{\mathbb{A}} f_{j} \mathrm{~d} \mu_{j \mid j-1}}{q_{j}}
$$

In addition, it can be verified that $f_{j}(x) \leq 1$ for almost every $x$ in $\mathbb{R}^{2} \times[0,2 \pi)$; otherwise 89$]$ would be violated.

Proof of (F1); Using the function $f_{j}$ obtained in Claim 2, let us define policies $\mathcal{P}_{k: N}$ as follows: For each $j$ in $\{k, \cdots, N\}$

$$
\mathcal{P}_{j}(x)= \begin{cases}0 & \text { with probability } f_{j}(x) \\ 1 & \text { with probability } 1-f_{j}(x)\end{cases}
$$

In conjunction with [92, we can verify that under the policies $\mathcal{P}_{k: N}$, the following holds:

$$
\mu_{j \mid j}(\mathbb{A})=\frac{\int_{\mathbb{A}} \mathbb{P}\left(\mathcal{P}_{j}\left(\mathbf{x}_{j}\right)=0 \mid \mathbf{x}_{j}=x\right) \mathrm{d} \mu_{j \mid j-1}}{q_{j}}
$$

Using (94) and Proposition D.12, it can be verified that the following hold for every $\mathbb{A}$ in $\mathfrak{B}$ :

$$
\mu_{j \mid j}(\mathbb{A})=\mathbb{P}\left(\mathbf{x}_{j} \in \mathbb{A} \mid \mathbf{R}_{k}=0, \cdots, \mathbf{R}_{j}=0\right)
$$

and

$$
q_{j}=\mathbb{P}\left(\mathbf{R}_{j}=0 \mid \mathbf{R}_{k}=0, \cdots, \mathbf{R}_{j-1}=0\right)
$$

subject to $\mathbf{R}_{j}=\mathcal{P}_{j}\left(\mathbf{x}_{j}\right)$ for all $j$ in $\{k, \cdots, N\}$. This completes the proof.

Henceforth, we make two additional claims under the policies $\mathcal{P}_{k: N}$ determined as in 93 to show that (F2) is true. 
Claim 3: For any Borel measurable subset $\mathbb{A}$ contained in $\overline{\mathbb{D}}_{j}^{c}$, it holds that

$$
\mathbb{P}\left(\mathbf{x}_{j} \in \mathbb{A} \mid \mathbf{R}_{k}=0, \cdots, \mathbf{R}_{j}=0\right)=0
$$

where the set $\overline{\mathbb{D}}_{j}$ is defined in $82 \mathrm{a}$.

Notice that

$$
\begin{aligned}
\mathbb{P}\left(\mathbf{x}_{j} \in \mathbb{A} \mid \mathbf{R}_{k}=0, \cdots, \mathbf{R}_{j}=0\right) & \mathbb{P}\left(\mathbf{x}_{j} \in \mathbb{A} \mid \mathbf{R}_{k}=0, \cdots, \mathbf{R}_{j-1}=0\right) \\
= & \frac{\mathbb{P}\left(\mathbf{R}_{j}=0 \mid \mathbf{R}_{k}=0, \cdots, \mathbf{R}_{j-1}=0\right)}{\mathbb{P}\left(\mathbf{x}_{j} \in \mathbb{A} \mid \mathbf{R}_{k}=0, \cdots, \mathbf{R}_{j}=1\right) \cdot \mathbb{P}\left(\mathbf{R}_{j}=1 \mid \mathbf{R}_{k}=0, \cdots, \mathbf{R}_{j-1}=0\right)} \\
- & \mathbb{P}\left(\mathbf{R}_{j}=0 \mid \mathbf{R}_{k}=0, \cdots, \mathbf{R}_{j-1}=0\right)
\end{aligned}
$$

To prove the claim, let $\mathbb{O}$ be an open set contained in $\overline{\mathbb{D}}_{j}^{c}$. By Lemma D.10 and Proposition D.12, we can derive the following:

$$
\mu_{j \mid j}^{(i)}(\mathbb{O})=\mu_{j \mid j}^{(i)}\left(\mathbb{O} \cap \overline{\mathbb{D}}_{j}^{(i)}\right) \leq \frac{\mu_{j \mid j-1}^{(i)}\left(\mathbb{O} \cap \overline{\mathbb{D}}_{j}^{(i)}\right)}{\mathbb{P}\left(\mathbf{R}_{j}^{(i)}=0 \mid \mathbf{R}_{k}^{(i)}=0, \cdots, \mathbf{R}_{j-1}^{(i)}=0\right)}
$$

where the set $\overline{\mathbb{D}}_{j}^{(i)}$ is defined in $83 \mathrm{a}$. By applying Theorem C.8 we can show that the following holds for all $i_{0}$ in $\mathbb{N}$ :

$$
\begin{aligned}
\mu_{j \mid j}(\mathbb{O}) & \leq \liminf _{i \rightarrow \infty} \mu_{j \mid j}^{(i)}(\mathbb{O}) \\
& \leq \liminf _{i \rightarrow \infty} \frac{\mu_{j \mid j-1}^{(i)}\left(\mathbb{O} \cap \overline{\mathbb{D}}_{j}^{(i)}\right)}{\mathbb{P}\left(\mathbf{R}_{j}^{(i)}=0 \mid \mathbf{R}_{k}^{(i)}=0, \cdots, \mathbf{R}_{j-1}^{(i)}=0\right)} \\
& \leq \liminf _{i \rightarrow \infty} \frac{\mu_{j \mid j-1}^{(i)}\left(\mathbb{O} \cap\left(\bigcup_{l \geq i} \overline{\mathbb{D}}_{j}^{(l)}\right)\right)}{\mathbb{P}\left(\mathbf{R}_{j}^{(i)}=0 \mid \mathbf{R}_{k}^{(i)}=0, \cdots, \mathbf{R}_{j-1}^{(i)}=0\right)} \\
& \leq \frac{\mu_{j \mid j-1}\left(\mathbb{O} \cap\left(\bigcup_{l \geq i_{0}} \overline{\mathbb{D}}_{j}^{(l)}\right)\right)}{\mathbb{P}\left(\mathbf{R}_{j}=0 \mid \mathbf{R}_{k}=0, \cdots, \mathbf{R}_{j-1}=0\right)}
\end{aligned}
$$

where (i) follows from Claim 1, (F1) and the fact that $\left\{\bigcup_{l \geq i} \overline{\mathbb{D}}_{j}^{(l)}\right\}_{i \in \mathbb{N}}$ is a decreasing sequence of measurable sets. Hence, from Lemma G.16, we have that

$$
\mu_{j \mid j}(\mathbb{O}) \leq \frac{\mu_{j \mid j-1}\left(\mathbb{O} \cap\left(\bigcap_{i \in \mathbb{N}} \bigcup_{l \geq i} \overline{\mathbb{D}}_{j}^{(l)}\right)\right)}{\mathbb{P}\left(\mathbf{R}_{j}=0 \mid \mathbf{R}_{k}=0, \cdots, \mathbf{R}_{j-1}=0\right)}=0
$$

Since $\overline{\mathbb{D}}_{j}^{c}$ is an open set, by selecting $\mathbb{O}=\overline{\mathbb{D}}_{j}^{c}$, we conclude that the following holds for every Borel measurable subset $\mathbb{A}$ of $\overline{\mathbb{D}}_{j}^{c}$ :

$$
\mu_{j \mid j}(\mathbb{A}) \leq \mu_{j \mid j}\left(\overline{\mathbb{D}}_{j}^{c}\right)=0
$$


Claim 4: Suppose that $\mathbb{P}\left(\mathbf{R}_{j}=1 \mid \mathbf{R}_{k}=0, \cdots, \mathbf{R}_{j-1}=0\right)$ is non-zero. Then, for any Borel measurable subset $\mathbb{A}$ contained in the set $\underline{\mathbb{D}}_{j}$ given in $82 \mathrm{~b}$, it holds that

$$
\mathbb{P}\left(\mathbf{x}_{j} \in \mathbb{A} \mid \mathbf{R}_{k}=0, \cdots, \mathbf{R}_{j}=1\right)=0
$$

To prove the claim, let $\mathbb{F}$ be a closed set contained in $\underline{\mathbb{D}}_{j}$. Notice that, by Lemma D.10, the following holds for all $i$ in $\mathbb{N}$ :

$$
\begin{aligned}
& \mathbb{P}\left(\mathbf{x}_{j} \in \mathbb{F} \cap \underline{\mathbb{D}}_{j}^{(i)} \mid \mathbf{R}_{k}^{(i)}=0, \cdots, \mathbf{R}_{j}^{(i)}=0\right) \\
& =\frac{\mathbb{P}\left(\mathbf{x}_{j} \in \mathbb{F} \cap \underline{\mathbb{D}}_{j}^{(i)} \mid \mathbf{R}_{k}^{(i)}=0, \cdots, \mathbf{R}_{j-1}^{(i)}=0\right)}{\mathbb{P}\left(\mathbf{R}_{j}^{(i)}=0 \mid \mathbf{R}_{k}^{(i)}=0, \cdots, \mathbf{R}_{j-1}^{(i)}=0\right)}
\end{aligned}
$$

Using (97) and Theorem C.8, we can show that the following holds for all $i_{0}$ in $\mathbb{N}$ :

$$
\begin{aligned}
\mu_{j \mid j}(\mathbb{F}) & \geq \limsup _{i \rightarrow \infty} \mu_{j \mid j}^{(i)}(\mathbb{F}) \\
& \geq \limsup _{i \rightarrow \infty} \mu_{j \mid j}^{(i)}\left(\mathbb{F} \cap \underline{\mathbb{D}}_{j}^{(i)}\right) \\
& =\limsup _{i \rightarrow \infty} \frac{\mu_{j \mid j-1}^{(i)}\left(\mathbb{F} \cap \underline{\mathbb{D}}_{j}^{(i)}\right)}{\mathbb{P}\left(\mathbf{R}_{j}^{(i)}=0 \mid \mathbf{R}_{k}^{(i)}=0, \cdots, \mathbf{R}_{j-1}^{(i)}=0\right)} \\
& \geq \limsup _{i \rightarrow \infty} \frac{\mu_{j \mid j-1}^{(i)}\left(\mathbb{F} \cap\left(\bigcap_{l \geq i} \mathbb{D}_{j}^{(l)}\right)\right)}{\mathbb{P}\left(\mathbf{R}_{j}^{(i)}=0 \mid \mathbf{R}_{k}^{(i)}=0, \cdots, \mathbf{R}_{j-1}^{(i)}=0\right)} \\
& \stackrel{(\mathbf{i})}{\geq} \frac{\mu_{j \mid j-1}\left(\mathbb{F} \cap\left(\bigcap_{l \geq i_{0}} \mathbb{D}_{j}^{(l)}\right)\right)}{\mathbb{P}\left(\mathbf{R}_{j}=0 \mid \mathbf{R}_{k}=0, \cdots, \mathbf{R}_{j-1}=0\right)}
\end{aligned}
$$

where (i) follows from Claim 1, (F1) and the fact that $\left\{\bigcap_{l \geq i} \mathbb{D}_{j}^{(l)}\right\}_{i \in \mathbb{N}}$ is an increasing sequence of measurable sets. Hence, from Lemma G.16, we have that

$$
\begin{aligned}
\mu_{j \mid j}(\mathbb{F}) & \geq \frac{\mu_{j \mid j-1}\left(\mathbb{F} \cap\left(\bigcup_{i \in \mathbb{N}} \bigcap_{l \geq i} \mathbb{D}_{j}^{(l)}\right)\right)}{\mathbb{P}\left(\mathbf{R}_{j}=0 \mid \mathbf{R}_{k}=0, \cdots, \mathbf{R}_{j-1}=0\right)} \\
& =\frac{\mu_{j \mid j-1}(\mathbb{F})}{\mathbb{P}\left(\mathbf{R}_{j}=0 \mid \mathbf{R}_{k}=0, \cdots, \mathbf{R}_{j-1}=0\right)}
\end{aligned}
$$

Using this relation, we can see that

$$
\begin{aligned}
& \mu_{j \mid j-1}(\mathbb{F}) \\
&= \mathbb{P}\left(\mathbf{x}_{j} \in \mathbb{F} \mid \mathbf{R}_{k}=0, \cdots, \mathbf{R}_{j-1}=0\right) \\
&= \mathbb{P}\left(\mathbf{x}_{j} \in \mathbb{F} \mid \mathbf{R}_{k}=0, \cdots, \mathbf{R}_{j}=0\right) \cdot \mathbb{P}\left(\mathbf{R}_{j}=0 \mid \mathbf{R}_{k}=0, \cdots, \mathbf{R}_{j-1}=0\right) \\
&+\mathbb{P}\left(\mathbf{x}_{j} \in \mathbb{F} \mid \mathbf{R}_{k}=0, \cdots, \mathbf{R}_{j}=1\right) \cdot \mathbb{P}\left(\mathbf{R}_{j}=1 \mid \mathbf{R}_{k}=0, \cdots, \mathbf{R}_{j-1}=0\right) \\
& \geq \mu_{j \mid j-1}(\mathbb{F}) \\
&+\mathbb{P}\left(\mathbf{x}_{j} \in \mathbb{F} \mid \mathbf{R}_{k}=0, \cdots, \mathbf{R}_{j}=1\right) \cdot \mathbb{P}\left(\mathbf{R}_{j}=1 \mid \mathbf{R}_{k}=0, \cdots, \mathbf{R}_{j-1}=0\right)
\end{aligned}
$$


Using the fact that $\mathbb{P}\left(\mathbf{R}_{j}=1 \mid \mathbf{R}_{k}=0, \cdots, \mathbf{R}_{j-1}=0\right)$ is non-zero, we obtain

$$
\mathbb{P}\left(\mathbf{x}_{j} \in \mathbb{F} \mid \mathbf{R}_{k}=0, \cdots, \mathbf{R}_{j}=1\right)=0
$$

Based on the closed regularity theorem (see Theorem 7.1.3 in [10]), we can see that the following holds for any Borel measurable set $\mathbb{A}$ contained in $\underline{\mathbb{D}}_{j}$ :

$$
\mathbb{P}\left(\mathbf{x}_{j} \in \mathbb{A} \mid \mathbf{R}_{k}=0, \cdots, \mathbf{R}_{j}=1\right)=0
$$

Proof of (F2); Recall that $\mathbb{E}_{\mathbf{x}_{j}}\left[J_{j}\left(\mathbf{x}_{j}, \mathcal{P}_{j: N}, \hat{x}_{j: N}^{\prime}\right) \mid \mathbf{R}_{k}=0, \cdots, \mathbf{R}_{j-1}=0\right]$ and $J_{j}^{*}$ are defined in (14) and [18), respectively, and that $\mathbf{R}_{j}=\mathcal{P}_{j}\left(\mathbf{x}_{j}\right)$ for all $j$ in $\{k, \cdots, N\}$. We will use the mathematical induction to show that the following is true:

$$
\mathbb{E}_{\mathbf{x}_{k}}\left[J_{k}\left(\mathbf{x}_{k}, \mathcal{P}_{k: N}, \hat{x}_{k: N}^{\prime}\right)\right]=\min _{\mathcal{P}_{k: N}^{\prime}} \mathbb{E}_{\mathbf{x}_{k}}\left[J_{k}\left(\mathbf{x}_{k}, \mathcal{P}_{k: N}^{\prime}, \hat{x}_{k: N}^{\prime}\right)\right]
$$

Using Claim 3 and Claim 4, we can derive the following:

$$
\begin{aligned}
& \mathbb{E}_{\mathbf{x}_{N}}\left[d^{2}\left(\mathbf{x}_{N}, \hat{x}_{N}^{\prime}\right) \mid \mathbf{R}_{N}=0\right] \\
& =\mathbb{E}_{\mathbf{x}_{N}}\left[\min \left\{d^{2}\left(\mathbf{x}_{N}, \hat{x}_{N}^{\prime}\right), c_{N}^{\prime}\right\} \mid \mathbf{R}_{N}=0\right] \\
& =\mathbb{E}_{\mathbf{x}_{N}}\left[J_{N}^{*}\left(\mathbf{x}_{N}, \hat{x}_{N}^{\prime}\right) \mid \mathbf{R}_{N}=0\right]
\end{aligned}
$$

and

$$
\begin{aligned}
c_{N}^{\prime} & =\mathbb{E}_{\mathbf{x}_{N}}\left[\min \left\{d^{2}\left(\mathbf{x}_{N}, \hat{x}_{N}^{\prime}\right), c_{N}^{\prime}\right\} \mid \mathbf{R}_{N}=1\right] \\
& =\mathbb{E}_{\mathbf{x}_{N}}\left[J_{N}^{*}\left(\mathbf{x}_{N}, \hat{x}_{N}^{\prime}\right) \mid \mathbf{R}_{N}=1\right]
\end{aligned}
$$

provided that $\mathbb{P}\left(\mathbf{R}_{N}=1 \mid \mathbf{R}_{k}=0, \cdots, \mathbf{R}_{N-1}=0\right)$ is nonzero. From (14), (18), and 98), we can derive that

$$
\begin{gathered}
\mathbb{E}_{\mathbf{x}_{N}}\left[J_{N}\left(\mathbf{x}_{N}, \mathcal{P}_{N}, \hat{x}_{N}^{\prime}\right) \mid \mathbf{R}_{N-1}=0\right] \\
=\mathbb{E}_{\mathbf{x}_{N}}\left[J_{N}^{*}\left(\mathbf{x}_{N}, \hat{x}_{N}^{\prime}\right) \mid \mathbf{R}_{N-1}=0\right]
\end{gathered}
$$

Suppose that the following relation holds:

$$
\begin{aligned}
& \mathbb{E}_{\mathbf{x}_{j+1}}\left[J_{j+1}\left(\mathbf{x}_{j+1}, \mathcal{P}_{j+1: N}, \hat{x}_{j+1: N}^{\prime}\right) \mid \mathbf{R}_{k}=0, \cdots, \mathbf{R}_{j}=0\right] \\
& =\mathbb{E}_{\mathbf{x}_{j+1}}\left[J_{j+1}^{*}\left(\mathbf{x}_{j+1}, \hat{x}_{j+1: N}^{\prime}\right) \mid \mathbf{R}_{k}=0, \cdots, \mathbf{R}_{j}=0\right]
\end{aligned}
$$

Then, using Claim 3 and Claim 4, we can derive the following:

$$
\begin{aligned}
& \mathbb{E}_{\mathbf{x}_{j}}\left[d^{2}\left(\mathbf{x}_{j}, \hat{x}_{j}^{\prime}\right)+\mathbb{E}_{\mathbf{x}_{j+1}}\left[J_{j+1}\left(\mathbf{x}_{j+1}, \mathcal{P}_{j+1: N}, \hat{x}_{j+1: N}^{\prime}\right) \mid \mathbf{x}_{j}\right] \mid \mathbf{R}_{k}=0, \cdots, \mathbf{R}_{j}=0\right] \\
& =\mathbb{E}_{\mathbf{x}_{j}}\left[\min \left\{d^{2}\left(\mathbf{x}_{j}, \hat{x}_{j}^{\prime}\right)+\mathbb{E}_{\mathbf{x}_{j+1}}\left[J_{j+1}^{*}\left(\mathbf{x}_{j+1}, \hat{x}_{j+1: N}^{\prime}\right) \mid \mathbf{x}_{j}\right], c_{j}^{\prime}\right\} \mid \mathbf{R}_{k}=0, \cdots, \mathbf{R}_{j}=0\right] \\
& =\mathbb{E}_{\mathbf{x}_{j}}\left[J_{j}^{*}\left(\mathbf{x}_{j}, \hat{x}_{j: N}^{\prime}\right) \mid \mathbf{R}_{k}=0, \cdots, \mathbf{R}_{j}=0\right]
\end{aligned}
$$


and

$$
\begin{aligned}
c_{j}^{\prime} & =\mathbb{E}_{\mathbf{x}_{j}}\left[\min \left\{d^{2}\left(\mathbf{x}_{j}, \hat{x}_{j}^{\prime}\right)+\mathbb{E}_{\mathbf{x}_{j+1}}\left[J_{j+1}^{*}\left(\mathbf{x}_{j+1}, \hat{x}_{j+1: N}^{\prime}\right) \mid \mathbf{x}_{j}\right], c_{j}^{\prime}\right\} \mid \mathbf{R}_{k}=0, \cdots, \mathbf{R}_{j}=1\right] \\
& =\mathbb{E}_{\mathbf{x}_{j}}\left[J_{j}^{*}\left(\mathbf{x}_{j}, \hat{x}_{j: N}^{\prime}\right) \mid \mathbf{R}_{k}=0, \cdots, \mathbf{R}_{j}=1\right]
\end{aligned}
$$

provided that $\mathbb{P}\left(\mathbf{R}_{j}=1 \mid \mathbf{R}_{k}=0, \cdots, \mathbf{R}_{j-1}=0\right)$ is non-zero. From (14), 18), and (101), we can derive that

$$
\begin{aligned}
& \mathbb{E}_{\mathbf{x}_{j}}\left[J_{j}\left(\mathbf{x}_{j}, \mathcal{P}_{j: N}, \hat{x}_{j: N}^{\prime}\right) \mid \mathbf{R}_{k}=0, \cdots, \mathbf{R}_{j-1}=0\right] \\
& =\mathbb{E}_{\mathbf{x}_{j}}\left[J_{j}^{*}\left(\mathbf{x}_{j}, \hat{x}_{j: N}^{\prime}\right) \mid \mathbf{R}_{k}=0, \cdots, \mathbf{R}_{j-1}=0\right]
\end{aligned}
$$

By induction, we conclude that 102 holds for all $j$ in $\{k, \cdots, N\}$. By Definition III.5 and the fact that

$$
\min _{\mathcal{P}_{k: N}^{\prime}} \mathbb{E}_{\mathbf{x}_{k}}\left[J_{k}\left(\mathbf{x}_{k}, \mathcal{P}_{k: N}^{\prime}, \hat{x}_{k: N}^{\prime}\right)\right]=\mathbb{E}_{\mathbf{x}_{k}}\left[J_{k}^{*}\left(\mathbf{x}_{k}, \hat{x}_{k: N}^{\prime}\right)\right]
$$

we conclude that the policies $\mathcal{P}_{k: N}$ belong to $\mathfrak{P}\left(\hat{x}_{k: N}^{\prime}\right)$.

\section{REFERENCES}

[1] Q. Du, V. Faber, and M. Gunzburger, "Centroidal voronoi tessellations: Applications and algorithms," SIAM Review, vol. 41, no. 4, pp. 637-676, 1999.

[2] A. Molin and S. Hirche, "An iterative algorithm for optimal event-triggered estimation," in 4th IFAC Conference on Analysis and Design of Hybrid Systems, June 2012, pp. 64-69.

[3] G. M. Lipsa and N. C. Martins, "Remote state estimation with communication costs for first-order LTI systems," IEEE Trans. Automat. Contr., vol. 56, no. 9, pp. 2013-2025, Sept 2011.

[4] A. Nayyar, T. Baar, D. Teneketzis, and V. V. Veeravalli, "Optimal strategies for communication and remote estimation with an energy harvesting sensor," IEEE Transactions on Automatic Control, vol. 58, no. 9, pp. 2246-2260, Sept 2013.

[5] S. Park and N. Martins, "Individually optimal solutions to a remote state estimation problem with communication costs," in Decision and Control (CDC), 2014 IEEE 53rd Annual Conference on, Dec 2014, pp. 4014-4019.

[6] Y. Xu and J. P. Hespanha, "Optimal communication logics in networked control systems," in Decision and Control, 2004. CDC. 43rd IEEE Conference on, vol. 4, Dec 2004, pp. 3527-3532.

[7] R. Cogill, S. Lall, and J. P. Hespanha, "A constant factor approximation algorithm for event-based sampling," in American Control Conference, 2007. ACC '07, July 2007, pp. 305-311.

[8] L. Li and M. Lemmon, "Performance and average sampling period of sub-optimal triggering event in event triggered state estimation," in Decision and Control and European Control Conference (CDC-ECC), 2011 50th IEEE Conference on, Dec 2011, pp. 1656-1661.

[9] L. Li, Z. Wang, and M. Lemmon, "Polynomial approximation of optimal event triggers for state estimation problems using SOSTOOLS," in American Control Conference (ACC), 2013, June 2013, pp. 2699-2704.

[10] R. M. Dudley, Real Analysis and Probability, 2nd ed. Cambridge University Press, 2002.

[11] B. T. McClintock, R. King, L. Thomas, J. Matthiopoulos, B. J. McConnell, and J. M. Morales, "A general discrete-time modeling framework for animal movement using multistate random walks," Ecological Monographs, vol. 82, no. 3, pp. 335-349, 2012.

[12] P. Billingsley, Probability and measure, 3rd ed. Wiley, 1995.

[13] A. V. Skorokhod, "Limit theorems for stochastic processes," Theory of Probability \& Its Applications, vol. 1, no. 3, pp. 261-290, 1956. 